Yang Bai, Song Han, Jing-Yu Guan, Jun Lin, Ming-Guang Zhao* and Guo-Biao Liang*

\title{
Contralateral C7 nerve transfer in the treatment of upper-extremity paralysis: a review of anatomical basis, surgical approaches, and neurobiological mechanisms
}

https://doi.org/10.1515/revneuro-2021-0122

Received September 9, 2021; accepted November 27, 2021;

published online January 3, 2022

\begin{abstract}
The previous three decades have witnessed a prosperity of contralateral C7 nerve (CC7) transfer in the treatment of upper-extremity paralysis induced by both brachial plexus avulsion injury and central hemiplegia. From the initial subcutaneous route to the pre-spinal route and the newly-established post-spinal route, this surgical operation underwent a series of innovations and refinements, with the aim of shortening the regeneration distance and even achieving direct neurorrhaphy. Apart from surgical efforts for better peripheral nerve regeneration, brain involvement in functional improvements after CC7 transfer also stimulated scientific interest. This review summarizes recent advances of CC7 transfer in the treatment of upper-extremity paralysis of both peripheral and central causes, which covers the neuroanatomical basis, the evolution of surgical approach, and central mechanisms. In addition, motor cortex stimulation is discussed as a viable rehabilitation treatment in boosting functional recovery after $\mathrm{CC} 7$ transfer. This knowledge will be beneficial towards improving clinical effects of CC7 transfer.
\end{abstract}

Keywords: brachial plexus avulsion injury; central hemiplegia; contralateral C7 nerve transfer; motor cortex stimulation; neuroplasticity.

\section{Introduction}

Brachial plexus injury (BPI) is a relatively rare, but nearly the most severe trauma, which mainly affects the youth in

\footnotetext{
*Corresponding authors: Guo-Biao Liang and Ming-Guang Zhao, Department of Neurosurgery, General Hospital of Northern Theater Command, No. 83 Wenhua Road, Shenhe District, Shenyang 110015, China, E-mail: liangguobiao6708@vip.163.com (G.-B. Liang), mingguangzhao1970@126.com (M.-G. Zhao)

Yang Bai, Song Han, Jing-Yu Guan and Jun Lin, Department of Neurosurgery, General Hospital of Northern Theater Command, No. 83 Wenhua Road, Shenhe District, Shenyang 110015, China
}

the prime of life. High-velocity motor vehicle accidents account for most cases undergoing surgical reconstruction, due to strong traction and stretch forces acting upon the brachial plexus when the patient falls on the shoulder, while other causes include high fall, penetrating injuries, and iatrogenic insults (Kaiser et al. 2020; Terzis and Kostopoulos 2007). According to the degree of injury, BPI could be classified into neurapraxia, axonotmesis, and neurotmesis (Seddon 1972). Neurotmesis could be further divided into nerve ruptures (postganglionic injury) and nerve avulsions (preganglionic injury) (Kachramanoglou et al. 2011). Global brachial plexus avulsion injury (BPAI) affecting all roots from $\mathrm{C} 5$ to $\mathrm{T} 1$ represents the most devastating injury of posttraumatic BPI. The victims suffer from a paralyzed and senseless upper-extremity, often with refractory neuropathic pain (Berman et al. 1998). The resultant disability inflicted in those patients is usually lifelong, which takes a heavy toll on individual physical health and wellbeing, and poses a tremendous burden to healthcare systems worldwide.

Surgical management for BPI includes neurolysis for nerve lesions in continuity, nerve repair and nerve grafting for lesions in discontinuity, and neurotization (or nerve transfer) for root avulsions (Kachramanoglou et al. 2011; Songcharoen 2008). The concept of nerve transfer is to sacrifice the function of a less-valued donor muscle to revive function in the recipient nerve and muscle that will undergo re-innervation (Narakas and Hentz 1988). Under the condition of global BPAI when intraplexus nerves are unavailable, extraplexus donor nerves remain as the sole choice for neurotization. Based on the neuroanatomical knowledge of peripheral nerves and the development of microsurgical techniques, various extraplexus donors were found, such as the accessory nerve, intercostal nerves, the anterior motor divisions of the cervical plexus, and phrenic nerve (Songcharoen 2008). Among these candidates, contralateral $\mathrm{C} 7$ nerve (CC7) emerges as the most promising donor source for BPI reconstruction, which provides a substantial number of axons for motoric and sensory restoration of the paralyzed upper-limb without greatly compromising the function of donor limb. Since the 
first CC7 transfer performed in Shanghai Huashan Hospital by (Gu et al. 1992), it has been widely used as a donor source for nerve transfer in brachial plexus reconstruction, especially for global BPAI.

Apart from BPI, central neurological injury (e.g. stroke, traumatic brain injury, and cerebral palsy) is another contributor to physical disability affecting both adults and children (Aisen et al. 2011; Hankey 2017; Rubiano et al. 2015). Although the mortality rate after central neurological injury has steadily decreased in the last few decades, the rate of incomplete recovery still remains high, especially for those with persistent upper-limb paralysis (Hankey 2017; Steyerberg et al. 2019). Typically, patients with unilateral brain injury exhibit contralateral spastic hemiplegia, which is characterized by spasticity, abnormal gestures, paresis of specific muscles, poor fine motor control, and sensory disturbances (Dobkin 2005). Unfortunately, current pharmacological and rehabilitative interventions have no certain effects, rendering a tough challenge to both neurologists and rehabilitation physicians (Synnot et al. 2017). In 2011, CC7 transfer was further introduced in the treatment of spastic arm paralysis by (Xu et al. 2011), which represents a paradigm change in the neurosurgical approach to the treatment of central hemiplegia.

Since the advent of CC7 transfer, nerve repair surgeons have been confronted by two major questions: the plateau in clinical efficacy of the original approach and a paucity of neurobiological underpinnings of CC7 transfer. A unanimous opinion has been reached that shortening the distance between the $\mathrm{CC} 7$ and the recipient nerve, or even achieving direct anastomosis, is crucial to nerve regeneration and subsequent functional improvements. Thus, hand surgeons are devoted to refining the technique by re-routing CC7, initially subcutaneously and later in the pre-spinal space (Zhang and $\mathrm{Gu}$ 2011). Recently, the post-spinal route was established by neurosurgeons, which also enabled direct neurorrhaphy in the treatment of central hemiplegia (Guan et al. 2019). Along with the innovations and improvements of the surgical approach is our preliminary understanding of cortical reorganization after CC7 transfer supported by clinical and preclinical evidence (Sun et al. 2014). This knowledge will provide a solid premise for the further refinement of this surgery and introduction of postoperative rehabilitation therapies.

This review summarizes recent breakthroughs and insights on CC7 transfer in the treatment of upper-extremity paralysis of peripheral and central causes. The neuroanatomical basis of CC7 transfer is firstly discussed. Next, we concentrate on the evolution process of the operative approach of $\mathrm{CC} 7$ transfer, and analyze the scope of application, operation essentials, and strengths and weaknesses of each passage from the perspective of surgery. Finally, current knowledge regarding cortical processing of functional recovery after $\mathrm{CC} 7$ transfer is summarized, on the basis of which the application prospect of motor cortex stimulation (MCS) in functional recovery after $\mathrm{CC} 7$ transfer is elaborated.

\section{Neuroanatomical basis of CC7 transfer}

\section{Rationale of CC7 transfer in the treatment of BPAI}

In the condition of BPAI, reconstructive surgery for the restoration of original neural connections is impossible since proximal nerve stumps are not available, rendering extraplexal neurotization as the main choice. The CC7 contains more than 23,000 myelinated nerve fibers (each division with more than 10,000 fibers), far exceeding the sum of other candidates including the phrenic nerve (3000), the accessory nerve (1600), the intercostal nerves (1200), as well as the recipient nerves such as the musculocutaneous nerve $(6000)$ and the median nerve $(18,000)$ (Gilbert et al. 2006). In addition, with the increased incidence of high-energy accidents, those with BPAI usually exhibit more extensive trauma, giving rise to a drastic decrease in available donor nerves for reinnervation. Considering these, the $\mathrm{CC} 7$ remains as the most appealing donor nerve owing to the capacity to provide enough nerve fibers.

Another premise for the selection of the $\mathrm{CC} 7$ is that dividing the $\mathrm{CC} 7$ would not cause permanent loss in sensory and motor functions in the healthy extremity. In terms of motor innervation, the $\mathrm{C} 5$ mainly forms the axillary nerve to innervate the deltoid muscle; the C6 mainly enters the musculocutaneous nerve to innervate the biceps; the C8 mainly passes within the median nerve to innervate the flexor digitorum profundus; and the T1 mainly forms the ulnar nerve to innervate intrinsic hand muscles. The $\mathrm{C} 7$, mainly by way of the radial nerve, nonspecifically contributes to the movements of the shoulder (the latissimus dorsi), the elbow (the triceps), the wrist (the extensor carpi radialis brevis), and the hand (the extensor digitorum) (Gu 1997; Terzis 1987; Wang et al. 2018). For brachial plexus nerve roots, the division of a single nerve root or even two nonneighboring roots did not permanently affect limb motor function in rodents, whereas the division of two neighboring nerve roots or three non-neighboring roots led to permanent motor dysfunction (Chen and $\mathrm{Gu}$ 2001). The decrease in grip and pinch strengths following $\mathrm{C} 7$ transection has been reported in clinical studies (Gu et al. 1992; Zhang and Gu 2011). 
However, this motor deficit is temporary since voluntary control over the muscles that the $\mathrm{C} 7$ innervates could be preserved due to compensation by adjacent nerve roots. For example, apart from $\mathrm{C} 7$ innervation, the latissimus dorsi is also innervated by the $\mathrm{C} 6$ and $\mathrm{C} 8$; the triceps by the $\mathrm{C} 5, \mathrm{C} 6$, $\mathrm{C} 8$, and $\mathrm{T} 1$; and the forearm extensors by the $\mathrm{C} 6$ and $\mathrm{C} 8(\mathrm{Gu}$ 1997; Gu and Shen 1994).

In terms of sensory function, the $\mathrm{C} 7$ comprises sensation in $<1 \%$ in the musculocutaneous nerve, $6 \%$ in the ulnar nerve, $16 \%$ in the radial nerve, and $19 \%$ in the median nerve (Li et al. 2014). Hence, when using the $\mathrm{C} 7$ as the donor, central sensory deficit will always be less than $25 \%$ in any given nerve, which is within the compensation ability of each nerve (Li et al. 2014). Clinical evidence showed that the patients usually experienced numbness and pain in the fingers in the first 3 months after surgery (Gu et al. 1992; Zhang and Gu 2011), and a very small portion of them $(0.71 \%)$ would develop refractory neuropathic pain after the harvest of the C7 (Li et al. 2015b). Taken together, the C7 nerve root may serve as a "spare nerve" in the highly structured and efficient nerve complex of the brachial plexus, and it is safe to sacrifice it for repair of target nerves.

The anterior division of $\mathrm{C}$, especially its anterior and medial part, contains a large portion of sensor fibers, while the posterior division mainly contains motor fibers, with no regularity in the distribution of sensory and motor fibers (Chuang et al.1998; Qin et al. 2012; Xu et al.1996). Therefore, partial CC7 as the donor was once recommended. There has been a shift in the transection plane from the entire root to the major root except the anterior and medial part of the anterior division (for retaining more sensation in the healthy limb), and to the anterior or the posterior division, with the posterior division used for restoring motor function or the anterior division used if sensory function is desired (Waikakul et al. 1999; Xu et al. 2004; Zhang and Gu 2011). Although selective transfer lessens sensory deficit and speeds up normal sensation recovery of the healthy extremity, the insufficiency of donor fibers would lead to permanent undesirable motoric improvements (Gao et al. 2010; Hierner and Berger 2007; Sammer et al. 2012; Tu et al. 2014). Thus, using the whole $\mathrm{C} 7$ is a better choice. To fully utilize the large source that $\mathrm{C} 7$ provides, it is recommended to prepare anterior and posterior divisions for repairing different recipient nerves (Liu et al. 2018; Terzis and Kokkalis 2009).

\section{Rationale of CC7 transfer in the treatment of spastic arm paralysis}

From the perspective of neuroanatomy, the muscle tension is mediated by spinal $y$-neuron circuitry, which itself is under descending inhibitory control of upper motor neurons (Kanning et al. 2010; Lemon 2008; Rudomin 1990). Under the condition of unilateral brain damage, the loss of descending control gives rise to contralateral flexor spasticity ( $\mathrm{Xu}$ et al. 2011). The impairment of cortical or subcortical motor pathways also leads to the loss of active movement (Lemon 2008; Welniarz et al. 2017), which is aggravated by the spasticity. Although contralesional hemisphere compensation is involved in the functional regain of limb control after unilateral brain injury (Lotze et al. 2006), the sparse connection between the contralesional hemisphere and the paralyzed hand in the contralesional side, i.e. the uncrossed anterior corticospinal tract (CST), limits the compensatory capacity (Jankowska and Edgley 2006; Ziemann et al. 1999). Based on this knowledge, we could infer that the release of spasticity and the restoration of brain motoric control over the limb are pivotal for the management of spastic hemiplegia.

For the treatment of upper-limb spasticity, selective peripheral neurotomy (SPN) and selective dorsal rhizotomy (SDR) are usually considered, which improve mobility and spasticity by interrupting peripheral inputs toward the over-activated spinal $y$-neuron circuitry (Aquilina et al. 2015; Enslin et al. 2019; Sindou et al. 2007). The former is the predominant procedure since spasticity is usually restricted to muscles subject to the control of a single or only a few peripheral nerves, while the latter is a valuable alternative to SPN for diffuse upper-limb spasticity (Maarrawi et al. 2006; Sindou and Mertens 1988). However, even with the release of flexor spasticity, the fine movement of upper extremity is still unlikely to restore since the extensor muscles do not have enough strength to antagonize that of the flexors (Xu et al. 2011). Thus, Xu et al. applied the concept of contralateral peripheral neurotization to improve the extensor function of the affected upper-extremity, and performed CC7 transfer to bridge the contralesional hemisphere and the contralesional paralyzed hand. Most patients exhibited flexor spasticity release and motor functional improvements, and regained self-care ability after surgery (Hua et al. 2015; Xu et al. 2011; Zheng et al. 2018). The reason for choosing ipsilesional C7 as the donor is the same as that described above, while the reason for selecting contralesional $\mathrm{C} 7$ as the recipient is that most C7 fibers diffusely innervate extensors (Gu 1997; Wang et al. 2018), which remain much weaker than flexors in stroke survivors in which the imbalance between flexors and extensors results in functional useless hand (Twitchell 1951). The division of the $C 7$ nerve at the affected side also weakens peripheral inputs toward spinal $y$-neuron circuit, thus reducing limb spasticity (Hua et al. 2016). 


\section{The evolution of operative approaches of CC7 transfer}

The donor source, interposition nerve graft, and effector muscles are the key peripheral factors determining the regenerative effect after nerve transfer. Owing to the determined neuroanatomical feature of $\mathrm{CC} 7$ and no effective strategy available for the irreversible muscular atrophy (Carraro et al. 2015; Midrio 2006), shortening the transfer path turns to be the sole factor influencing nerve regeneration (Bertelli et al. 2005), which becomes the paramount research direction for nerve repair surgeons. Correspondingly, there have been a series of interesting changes in the operative approach since the establishment of CC7 transfer. Generally, operative approaches for $\mathrm{CC} 7$ transfer could be categorized as pretracheal routes (including the original and the modified subcutaneous routes, the retrosternocleidomastoid route, and the carotid sheath route), pre-spinal routes (including the original and the modified

Table 1: A summary of operative approaches for $\mathrm{CC} 7$ transfer.

\begin{tabular}{|c|c|c|c|}
\hline Operative approaches & Subject & $\begin{array}{l}\text { Harvesting level of donor } \\
\text { nerves }\end{array}$ & $\begin{array}{l}\text { Targeting nerves and the length of Advantages and disadvantages } \\
\text { bridging nerves }\end{array}$ \\
\hline
\end{tabular}

Pre-tracheal routes

Original subcutaneous route

Gu et al. 1992
49 patients At the common trunk; at the with BPAI origin of the posterior division or anterior division
10 patients At the distal ends of its both with BPAI anterior and posterior divisions

Peng et al. (2003)

Modified subcutaneous route

Feng et al. (2010)

Carotid sheath route Doshi and Bhatt (2016)
10 patients At the distal ends of both with BPAl divisions
Four pa- At the distal ends of both tients with divisions BPAI
The ulnar nerve graft or sural donor nerves $(\geq 18 \mathrm{~cm})$ for repairing the radial nerve, the musculocutaneous nerve, the median nerve, the thoracodorsal nerve, or the musculocutaneous nerve

Sural nerve grafts with mean distance of $7.8 \mathrm{~cm}$ for repairing the upper or the lower trunk of the injured plexus Direct neurorrhaphy achieved in two patients with C8-T1 roots repaired; a $4.5-\mathrm{cm}$ nerve graft used in others with anterior division of the lower trunk connected Direct neurorrhaphy with the anterior division of the lower trunk
Disadvantages: Delayed motoric recovery owing to long regeneration distance, two neurorrhaphy sites, and insufficient use of the donor nerve; complete functional loss of the ulnar nerve; a long incision Advantages: Full use of the donor; shorter nerve grafts; and avoiding subcutaneous hematoma ${ }^{b}$

Advantages: Full use of the donor; shorter nerve grafts ${ }^{b}$

Advantages: Direct coaptation ${ }^{b}$ Disadvantages: Risk of vagal nerve injury and sensory disorder in the extremity during vascular pulsation ${ }^{\text {b }}$

\section{Pre-spinal routes}

Original pre-spinal route

McGuiness and Kay (2002)

Modified pre-spinal route

Wang et al. (2003)

Modified pre-spinal route Xu et al. (2007)
One pa-

The posterior division of

BPAI

CC7

13 patients At the distal ends of both with BPAI divisions

The median nerve repaired with the ulnar nerve graft

Nerve grafts with a length of $9.4 \pm 1.2 \mathrm{~cm}$ for repairing the upper trunk or both divisions of the upper trunk

Eight pa- $\quad$ At the trunk division level or Grafts for repairing the supratients with the division cord level BPAI clavicular and infraclavicular brachial plexus were

$6.25 \pm 0.35 \mathrm{~cm}$ and $8.56 \pm 0.45 \mathrm{~cm}$, respectively, with $\mathrm{CC} 7$ cut at the trunk division level; when $\mathrm{CC} 7$ transected at the division cord level, no graft was used for repairing $\mathrm{C} 5-\mathrm{C} 6$ roots $(N=2)$, and a $3-\mathrm{cm}$ graft used for repairing the upper trunk $(N=1)$

Direct neurorrhaphy achieved in bilateral $\mathrm{C} 7$ nerves
Advantages: Simpler dissection; shorter nerve grafts; and protected placement of the graft ${ }^{\mathrm{b}}$ Disadvantages: Potential injury to important structures, including vertebral vessels, the thoracic duct, and the recurrent compression upon esophageal motility $^{b}$ laryngeal nerve; potential CC7
One patient At the distal ends of both with spastic divisions arm 
Table 1: (continued)

\begin{tabular}{|c|c|c|c|}
\hline Operative approaches & Subject & $\begin{array}{l}\text { Harvesting level of donor } \\
\text { nerves }\end{array}$ & $\begin{array}{l}\text { Targeting nerves and the length of Advantages and disadvantages } \\
\text { bridging nerves }\end{array}$ \\
\hline
\end{tabular}

\section{Vertebral routes}

Vertebral body route

Wang et al. (2009)

15 At the distal ends of both

cadavers divisions

Direct neurorrhaphy achieved in

bilateral $\mathrm{C} 7$ nerves

Intervertebral disc route

10

Vanaclocha et al. (2015)

cadavers

At the distal ends of both divisions

Direct neurorrhaphy achieved in bilateral $\mathrm{C} 7$ nerves

At the distal ends of both divisions

At the distal ends of both divisions tient with spastic arm

\section{Post-spinal routes}

Modified post-spinal route

Guan et al. (2019)

Posterior intradural route ${ }^{\mathrm{a}}$ One

Jiang et al. (2019)

cadaver

C7 nerve root on one side divided as distally as possible, while $\mathrm{CC} 7$ root divided as proximally as possible within the subdural space
Nerve grafts with a length of $1.0 \mathrm{~cm}$ for repairing the upper trunk, or $3.0 \mathrm{~cm}$ for repairing the lower trunk Direct neurorrhaphy achieved in bilateral C7 nerves
The length of sural nerve graft was about $1 \mathrm{~cm}$ connecting posterior divisions of both $\mathrm{C} 7$, and about $1.5-2 \mathrm{~cm}$ connecting anterior divisions of both $\mathrm{C} 7$
Posterior extradural route $^{\mathrm{a}}$ Nine Yang et al. (2020) cadavers
CC7 nerve root was divided Direct neurorrhaphy achieved in at the junction of the VR and bilateral DRs as well as bilateral $D R$, while ipsilateral $C 7$ root VRs of $C 7$ nerves sectioned at the nerve root outlet of the dural mater
Disadvantages: Local hematoma; injury to adjacent structures; nerve compression within the tunnel ${ }^{b}$

Disadvantages: $\mathrm{CC} 7$ compression within the disc space; spine instability; potential injury to the vertebral/radicular artery ${ }^{\mathrm{b}}$

Disadvantages: Nerve graft still needed; shift in operative position $^{\text {b }}$

Advantages: Avoiding potential injury to key structures in the pre-spinal route

Disadvantages: Shift in position; spine instability; potential nerve traction during neck movement $^{c}$

Advantages: Multiple anastomose enables full use of the donor $\mathrm{C} 7$ and precise repair ${ }^{\mathrm{C}}$ Disadvantages: Spinal instability; complications after opening the dural sac; potential compression of the spinal cord within the subarachnoid space; weak anti-tension strength of anastomosed nerves; difficulty in identifying the VRs and the DRs; uncertain reinnervation owing to pre-ganglionic neurorrhaphy ${ }^{c}$

Advantages: Direct, precise and tension-free coaptation of bilateral C7 nerves; avoiding complications after opening the dural sac; no obvious compression of spinal cord within the spinal canal ${ }^{c}$

Disadvantages: Spinal instability; uncertain reinnervation owing to pre-ganglionic neurorrhaphy ${ }^{c}$

andicate operative approaches of CC7 transfer designed for the treatment of spastic limb paralysis. ${ }^{b}$ Compared with the original approach of CC7 transfer designed for the treatment of BPAI, i.e. the original subcutaneous route by Gu et al. ${ }^{\circ} \mathrm{Compared}$ with the original approach of CC7 transfer designed for the treatment of spastic limb paralysis, i.e., Huashan pre-spinal route by $\mathrm{Xu}$ et al.

pre-spinal routes, and Huashan pre-spinal route), vertebral routes (including the vertebral body route and the intervertebral disc route), post-spinal routes (including the original and the modified post-spinal routes), and intraspinal routes (including the posterior intradural route and the posterior extradural route) (Table 1). 


\section{The original subcutaneous route}

A subcutaneous tunnel anterior to the neck and pectoral region was utilized in the original approach for $\mathrm{CC} 7$ transfer (Figure 1A, B) (Gu et al. 1992). The ulnar nerve in the affected side was usually sacrificed as the bridging nerve inasmuch as intrinsic hand muscles were unlikely to recover after BPAI. Additionally, sural nerves in groups of three or four strands could also be used. When using the ulnar nerve as graft, the $\mathrm{CC} 7$ was severed at the common trunk or the origin of the anterior/posterior division through a supraclavicular transverse incision. Then, the ulnar nerve severed at the wrist was dissected toward the axilla at the level just below the entrance of superior ulnar collateral artery. The reversed ulnar neurovascular bundle was transposed through a pectoral subcutaneous tunnel with a length of at least $18 \mathrm{~cm}$ to connect the proximal end of $\mathrm{CC} 7$. The second procedure was performed when nerve regeneration reached the axilla as judged by clinical and physiological examinations (usually 10 months). Then, the proximal end of the ulnar nerve was cut and anastomosed to recipient nerves, including the radial nerve or the median nerve (Gu et al. 1992; Xu et al. 2008; Zhang and $\mathrm{Gu}$ 2011).

The chief pitfall of this method is the long delay of manifestation of motoric recovery caused by the long distance the regenerating axons have to travel along the graft as well as the two neurorrhaphy sites the axons have to cross to reach the motor endplates of forearm muscles (Yang et al. 2015). Additionally, only the anterior or the posterior division of $\mathrm{CC} 7$ is used owing to the great incongruence between the number of $\mathrm{CC} 7$ fibers and that of terminal recipient nerves, which leads to a waste of the donor source. Moreover, the employment of the ulnar nerve results in complete loss of function in its innervation areas as well as a long incision (about $36 \mathrm{~cm}$ ) on the injured upper extremity (Waikakul et al. 1999). Considering these, the establishment of the shortest, safe, and effective way tailored to regional anatomy for achieving direct coaptation will inevitably improve the surgical outcomes.

\section{The retrosternocleidomastoid route}

In 2003, Peng et al. modified the original route via a tunnel under the sternocleidomastoid muscle for repair of root and trunk injuries (Figure 1C). Several improvements were made to shorten the nerve gap. For preparation of bilateral plexus nerves, the CC7 was dissected to the middle trunk as far as its anterior and posterior divisions and transected at the distal end to the division to increase the length of donor nerves, with the upper or the lower trunk of the injured plexus designated as the recipient instead of terminal branches. For
A

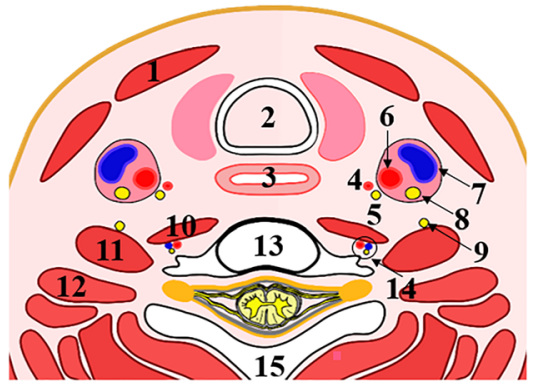

C

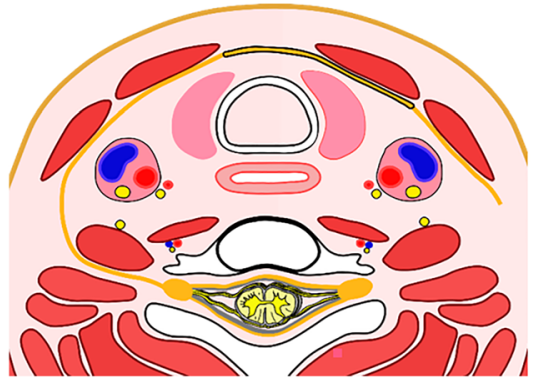

B

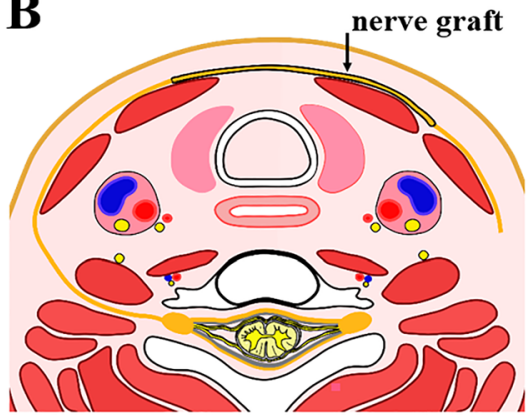

D

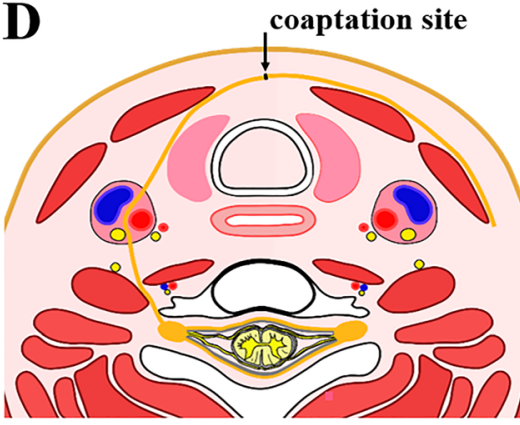

Figure 1: Schematic diagrams of pre-tracheal routes. (A) A representative axial section through the $C 7$ vertebral body showing normal cervical anatomical structures. (B-D) Diagrams of the subcutaneous route (B), the retrosternocleidomastoid route (C), and the carotid sheath route (D). (1) sternocleidomastoid muscle, (2) trachea, (3) esophagus, (4) superior thyroid artery, (5) recurrent laryngeal nerve, (6) common carotid artery, (7) common jugular vein, (8) vagus nerve, (9) phrenic nerve, (10) longus collis muscle, (11) anterior scalene muscle, (12) middle scalene muscle, (13) C7 vertebral body, (14) vertebral artery, vertebral vein and sympathetic trunk, and (15) spinous process. 
modification of the passage, a tunnel connecting bilateral nerve stumps was constructed behind bilateral sternocleidomastoid muscles and in front of the trachea by blunt dissection while protecting the carotid sheath. If necessary, bilateral anterior scalenus muscles could be severed to further shorten the distance (Peng et al. 2003).

During surgery, the authors observed that the mean distance between the nerve stumps was $7.8 \mathrm{~cm}$ (range: $5.5-10.0 \mathrm{~cm}$ ), so a nerve graft was used for bridging the gap. Compared with the subcutaneous tunnel, this modified pathway necessitates a short free nerve instead of the long vascularized ulnar nerve graft, and avoids the risk of subcutaneous hematoma. Results of 15-month follow-up showed that four of six patients with repair of the upper trunk obtained elbow flexion and shoulder abduction, while one patient with the lower trunk repaired gained pain sensation in the wrist and exhibited motor nerve action potentials in the median nerve and the ulnar nerve (Peng et al. 2003). Recently, (Wang et al. 2018) repaired the middle trunk of injured plexus via this route with the average nerve graft of $9.5 \mathrm{~cm}$, which was proven to be safe and effective in rescuing shoulder adduction and upper-extremity extension in this patient series.

\section{The modified subcutaneous route}

In 2010, Feng et al. reported the subcutaneous route to repair the lower trunk of the injured plexus for gaining better hand function. The preparation of anterior and posterior divisions of the CC7 was the same as the way described in the retrosternocleidomastoid route. After severing the posterior division of the lower trunk and other affiliated nerves, the rest part of the injured C8-T1 nerve roots or the lower trunk was passed from the incision in the injured infraclavicular area to the sternoclavicular joint on the uninjured side through the subcutaneous passage across the anterior surface of the neck and chest. Direct neurorrhaphy was achieved on the C8-T1 residual nerve roots in two patients, while a $4.5-\mathrm{cm}$ nerve graft was used in another two patients with the anterior division of the lower trunk connected. Results of 26-month follow-up showed that the patients gained favorable wrist flexion, finger flexion, and hand sensation (Feng et al. 2010).

This modified approach shortens the length of nerve graft in comparison to the traditional approach and avoids humerus shortening (Yu et al. 2003). By transferring CC7 to C8-T1 roots, both the median nerve and the ulnar nerve would be subject to reinnervation, which theoretically guarantees better function recovery than repairing a single terminal nerve. The overall cross-sectional area of both divisions of the $\mathrm{CC} 7$ matches well with that of the C8-T1 roots or the lower trunk, which enables the maximum use of the donor (Feng et al. 2019b). However, this method fails to achieve direct neurorrhaphy in all cases, so it may be preferred for repair of lower-level root injuries.

\section{The carotid sheath route}

In 2016, Doshi et al. reported the carotid sheath route for direct repair of the anterior division of the lower trunk (Figure 1D). When performing the pre-spinal route, they noted that the mobilized lower trunk (with the posterior division severed), which reached the level of the anterior scalenus muscle by route deep to the clavicle, could also be transferred to the suprasternal notch when brought out from below the clavicle. Thus, direct anastomose could be achieved with $\mathrm{CC} 7$ brought to the midline. Finally, a safe passage was constructed through the carotid sheath, with the common carotid artery medially and the internal jugular vein laterally. The $\mathrm{CC} 7$ dissected medial to the anterior scalenus muscle could be directly transferred along this path to the subcutaneous plane at the suprasternal notch. Direct approximation of the $\mathrm{CC} 7$ to the lower trunk was attempted in all the 10 patients with the aid of arm adduction, but the clinical outcome of this route, i.e. the recovery of extrinsic finger flexion, remains to be observed (Doshi and Bhatt 2016).

Since vertebral vessels, inferior thyroid vessels, the sympathetic chain, and the recurrent laryngeal nerve lie medial and deep to the common carotid artery, this route could avoid injury to these structures. Considering these, this route was recommended by the authors as a substitute for pre-spinal routes when repairing the lower trunk (Doshi and Bhatt 2016). However, the major risk is vagal nerve injury and subsequent cardiac and other visceral disorders, since the vagal nerve is located inside the carotid sheath. Another potential complication is the occurrence of sensory disorders in the extremity during palpation of the carotid artery.

\section{The original pre-spinal route}

In 2002, Mcguiness et al. reported one case of CC7 transfer accomplished by passing the nerve through the pre-spinal and retropharyngeal space to repair the median nerve in the treatment of obstetric brachial plexus palsy (Figure 2A, B). With a curved incision in the neck, the authors retracted the carotid sheath and sternomastoid muscles laterally and the esophagus and trachea medially to form a route through the retropharyngeal space anterior to the prevertebral fascia. A similar maneuver from the recipient side allows completion 
A

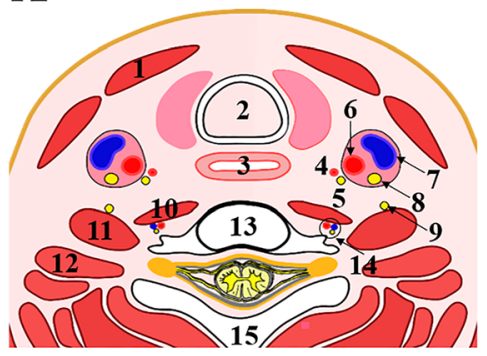

D

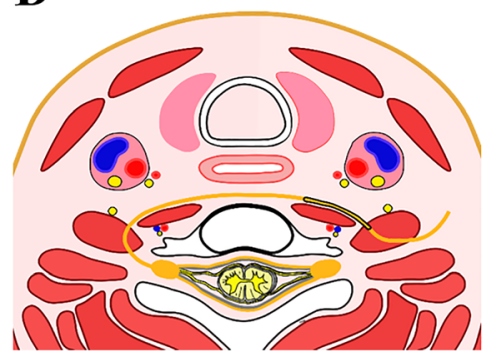

B

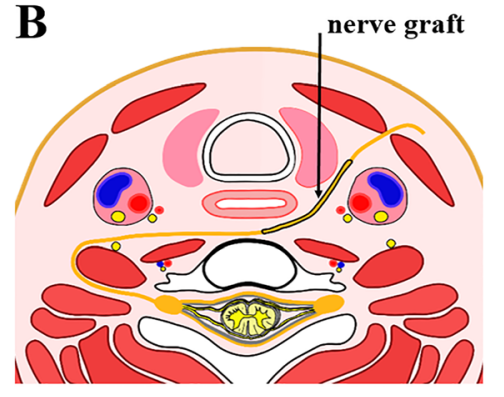

$\mathbf{E}$

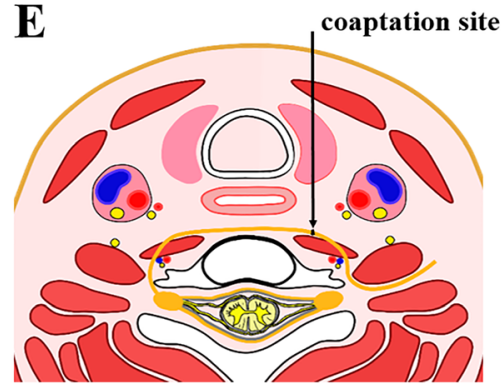

C

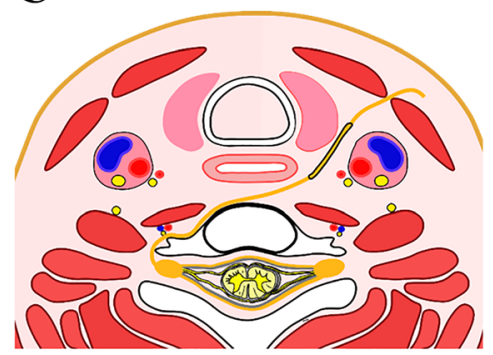

Figure 2: Schematic diagrams of pre-spinal routes. (A) A representative axial section through the $C 7$ vertebral body showing normal cervical anatomical structures. (B-E) Diagrams of the original pre-spinal route by Mcguiness et al. (B), modified pre-spinal routes by Wang et al. (C) and by Xu et al. (D), and Huashan pre-spinal route (E). The anatomical structures marked by 1-15 are the same as those described in Figure 1.

of the tunnel and subsequent anastomosis of CC7 and the ulnar nerve graft. Important structures lying behind the tunnel include longus colli muscles, anterior scalenus muscles, and the phrenic nerve. Therefore, much more focus should be paid to preserve the phrenic nerve on the surface of anterior scalenus muscles. Comparing with the subcutaneous tunnel, this route has merits of a simpler dissection, a shorter distance, and protected placement of the graft (McGuiness and Kay 2002).

\section{The modified pre-spinal route}

Nearly at the same time when Mcguiness et al. reported the pre-spinal route, Wang et al. reported another pre-spinal route for $\mathrm{CC} 7$ transfer in a Chinese journal concerning hand surgery (Figure $2 \mathrm{C}$ ). In this route, the $\mathrm{CC} 7$ was transferred underneath the anterior scalenus muscle, the vertebral artery, and the longus colli muscle, and then passed through the retro-esophageal space to neurotize the recipient nerve. Much more focus should be paid to the protection of vertebral vessels when establishing the tunnel underneath the anterior scalenus muscles (Wang et al. 2003, 2004, 2013; Wang and Xue 2014). In 2017, the authors reviewed the clinical outcomes of 200 patients with the upper or the lower trunk repaired via this route in their institution. Direct coaptation with the upper trunk was achieved in three of the total 60 patients, while interposition sural nerve graft with a length of $6.8 \pm 1.7 \mathrm{~cm}$ was used in other cases. Direct coaptation with the lower trunk was accomplished in all the 140 cases by means of humeral shortening osteotomy (74 cases) and extended mobilization of the lower trunk. In addition, immobilization of the injured upper-extremity in a specific position was necessitated to minimize the tension of anastomosis after operation (Yang et al. 2017). Unlike Mcguiness's method, Wang tried to achieve direct coaptation or utilize shorter nerve grafts instead of a long vascularized ulnar nerve. Although immobilizing the injured upper extremity in a specific position facilitated a tension-free direct coaptation, the tension across the neurorrhaphy site may occur after removal of the brace and postoperative shoulder movement (Wang et al. 2013). Thus, the decision of direct coaptation should be made with great caution.

In 2007, Xu et al. introduced another alternative passage to the Mcguiness method by transferring CC7 through the pre-spinal space with bilateral anterior scalenus muscles severed (Figure 2D) in eight cases of BPAI. After bilateral exposure of the brachial plexus, the phrenic nerve on the surface of the anterior scalenus muscle was released and protected, with the $\mathrm{C} 7$ vertebral body as a landmark. After severing the anterior scalenus muscle, the $\mathrm{CC} 7$ was flipped in the posteromedial direction of the phrenic nerve, and a tunnel was developed between the anterior vertebral body and the posterior surface of the esophagus by 
bilateral mobilization. In the first five patients, the $\mathrm{CC} 7 \mathrm{cut}$ at the trunk division level (averaged length of $4.67 \mathrm{~cm}$ ) reached one-third of the vertebral body on the injured side. The nerve graft for repairing the supraclavicular and infraclavicular brachial plexus was $6.25 \pm 0.35 \mathrm{~cm}$ and $8.56 \pm 0.45 \mathrm{~cm}$, respectively. In the last three cases with the harvested CC7 transected at the division cord level for obtaining more length (averaged length of $6.85 \mathrm{~cm}$ ), direct neurorrhaphy was achieved to the residual $\mathrm{C} 5$ and $\mathrm{C} 6$ roots in two cases, and a 3-cm nerve graft was used to repair the upper trunk in one case. This approach possesses several advantages. This route involves the exposure of the anterior space of the vertebral body instead of the carotid sheath or other important structures in the neck. Additionally, the transection of the anterior scalenus muscle avoids potential compression of the transferred nerve and further reduces the distance of nerve regeneration. All these merits allow the $\mathrm{CC} 7$ to be transferred more smoothly and safely via the pre-spinal route (Xu et al. 2007, 2008).

\section{Huashan pre-spinal route}

Unlike those with BPI, patients with central neurological injury have intact peripheral nerves, which adds to the possibility of direct anastomosis of bilateral $\mathrm{C} 7$ nerves. After a long-term exploration, $\mathrm{Xu}$ et al. determined the shortest "Huashan pre-spinal route" for CC7 transfer by severing bilateral anterior scalenus muscles, penetrating the longus colli muscle to establish a nerve transfer tunnel, and accomplishing bilateral $\mathrm{C} 7$ anastomosis via the prevertebral space (Figure 2E). A key point of this route is to protect the vertebral artery. Anatomical measurements showed that the total tilt angel of the vertebral artery to the sagittal plane was $25.5 \pm 4.5^{\circ}$. Hence, it is strongly recommended that the longus colli muscle be penetrated at the outer two-thirds point with forceps at a tilt angle of above $35^{\circ}$ to transfer $\mathrm{C} 7$ on the outside of the vertebral artery. The transection of anterior scalenus muscles provides adequate exposure and acquisition of fulllength $\mathrm{C} 7$ nerve, while the tunnel through the long colli muscle allows for nerve transfer through a shorter path. All these merits made direct anastomosis of bilateral C7 possible in most cases, with the exception for a few cases with particularly short $\mathrm{C} 7$ necessitating nerve grafting ( $\mathrm{Li}$ et al. 2020; Xu 2020).

However, there are several concerns regarding CC7 transfer in the treatment of central hemiplegia. Firstly, these studies had a relatively small sample size and have not yet been replicated in other countries, only with a similar study from China at single case level (Pan et al. 2020). Therefore, there is a pressing need for the validation of these studies in large multicenter cohorts. Secondly, the patients included in the studies were relatively young. Whether this surgery takes effects in the treatment of stroke, which is more prevalent in the elderly, remains to be investigated (Alawieh et al. 2021). Thirdly, the pre-spinal route presents a high demand on operation skills and confers a high risk of injury to important structures, such as vertebral vessels, the thoracic duct, the recurrent laryngeal nerve, the phrenic nerve, the sympathetic trunk, the inferior thyroid artery, and the esophagus. In addition, the potential $\mathrm{C} 7$ nerve compression caused by esophageal motility induces sensory abnormality of the donor upper-extremity and hampers nerve regeneration. (Li et al. 2015b) reviewed the complications related to the modified pre-spinal route in 425 patients with BPAI. Complications associated with the pre-spinal tunnel included vertebral artery laceration in two cases, temporary recurrent laryngeal nerve palsy in five cases, pain and numbness in the healthy upper-extremity during swallowing in four cases, and postoperative brainstem thrombosis in one case. Hence, the prevention of these complications is necessary to popularize this procedure.

\section{The vertebral body route}

In 2009, Wang et al. established the vertebral channel route in adult cadavers (Figure 3A, B) based on the common sense that a straight line is the shortest between two points. Considering the upper and the lower trunks of brachial plexus are roughly located at the level of the upper and lower edges of $\mathrm{C} 7$ vertebral body, respectively, the authors selected the $\mathrm{C} 7$ vertebral body for construction of nerve transfer tunnel. After exposure of the $\mathrm{C} 7$ vertebral body through the supraclavicular transverse incision, a hole was drilled horizontally at the posterior edge of $\mathrm{C} 7$ vertebral body antero-inferiorly to $\mathrm{C} 7$ intervertebral foramen. Then, the mobilized $\mathrm{C} 7$ root was flipped medially and posteriorly to the vertebral artery towards the contralateral side through the hole. Direct coaptation was achieved between the $\mathrm{C} 7$ root and contralateral upper or lower trunk in all the 30 cases. Anatomical measurements showed that the distance between the $\mathrm{C} 7$ root and contralateral upper trunk through the vertebral body route, the pre-spinal route, and the subcutaneous route was $6.97 \pm 0.56 \mathrm{~cm}, 10.04 \pm 0.94 \mathrm{~cm}$, and $16.56 \pm 1.24 \mathrm{~cm}$, respectively, with statistical significance among them $(P<0.01)$; the distance between the $\mathrm{C} 7$ root and the contralateral lower trunk through these routes mentioned above was $6.82 \pm 0.92 \mathrm{~cm}, 9.91 \pm 0.83 \mathrm{~cm}$, and $17.64 \pm 0.97 \mathrm{~cm}$, also with significant difference, suggesting that the vertebral route is the shortest way for CC7 transfer (Wang et al. 2009). To minimize physical impacts on the spine, the diameter of the hole was kept around $6 \mathrm{~mm}$, just allowing the $\mathrm{C} 7$ nerve (with 
an averaged diameter of $5.63 \mathrm{~mm}$ ) transferring through the vertebral body. This hole approximately destroyed $1 / 3$ of the lateral bony cortex, with the majority of anterior cortex remaining intact. Vertebral construction test showed that the maximum load of $\mathrm{C} 7$ vertebral body was decreased by only $14.89 \%$ compared with control group, with no significant difference $(P>0.05)$, suggesting no impact of drilling on the biomechanical properties of the vertebral body. In addition, anatomical measurements showed that the relative displacement between the $\mathrm{C} 7$ and $\mathrm{T} 1$ vertebral bodies did not result in the compression or stretching of the transferred nerve (Xia et al. 2010). Despite all these merits, other major concerns, such as punching-related local hematoma, surgical damage to adjacent structures, and nerve compression within the tunnel caused by vertebral remodeling, restrict its clinical usefulness.

\section{The intervertebral disc route}

In 2015, Vanaclocha et al. reported an intervertebral disc channel for $\mathrm{CC} 7$ transfer in a cadaveric study (Figure 3C). After the exposure of $\mathrm{C} 7$ vertebral body, $\mathrm{C} 6-\mathrm{C} 7$ discectomy was performed, with bilateral longus colli muscles preserved to avoid injury to the sympathetic chain. Then, the donor $\mathrm{C} 7$ was freed from its ligamentous attachments, and rotated through the disc space to reach the contralateral side. An autologous iliac crest bone graft was used to keep the disc space open, with enough space left between the graft and the spinal cord for transfer of CC7. The use of cervical plate was also recommended to strengthen spinal stability. During the surgery, they observed that when the CC7 was transferred rounding the vertebral artery into the retropharyngeal space, the root got past the middle line $2.4 \pm 1.3 \mathrm{~cm}$, and re-routing CC7 medial to the artery further gained only $1.2 \pm 0.3 \mathrm{~cm}$. Thus, interposing graft nerves were needed for the pre-spinal route. However, transdiscal CC7 transfer further increased the length available by $5.3 \pm 1.2 \mathrm{~cm}$, thus enabling direct tension-free coaptation of CC7 with the C8-T1 roots in all the 10 cases. However, several limitations restrict its clinical usefulness. Firstly, the overgrowth of bone graft might compress the $\mathrm{CC} 7$ placed behind it in the disc space. Secondly, fusing the cervical disc may influence the dynamics of the spine. Thirdly, the mobilization and transfer of the CC7 puts the vertebral artery and the radicular artery at risk (Vanaclocha et al. 2015).

\section{The original post-spinal route}

To seek for alternatives to the pre-spinal route, Xiang et al. explored the feasibility of a posterior spinal route for CC7 transfer in a cadaveric study in 2012 (Figure 3D). The posterior cervical area is devoid of important vessels and nerves. In addition, the width of the interspinous space of lower cervical
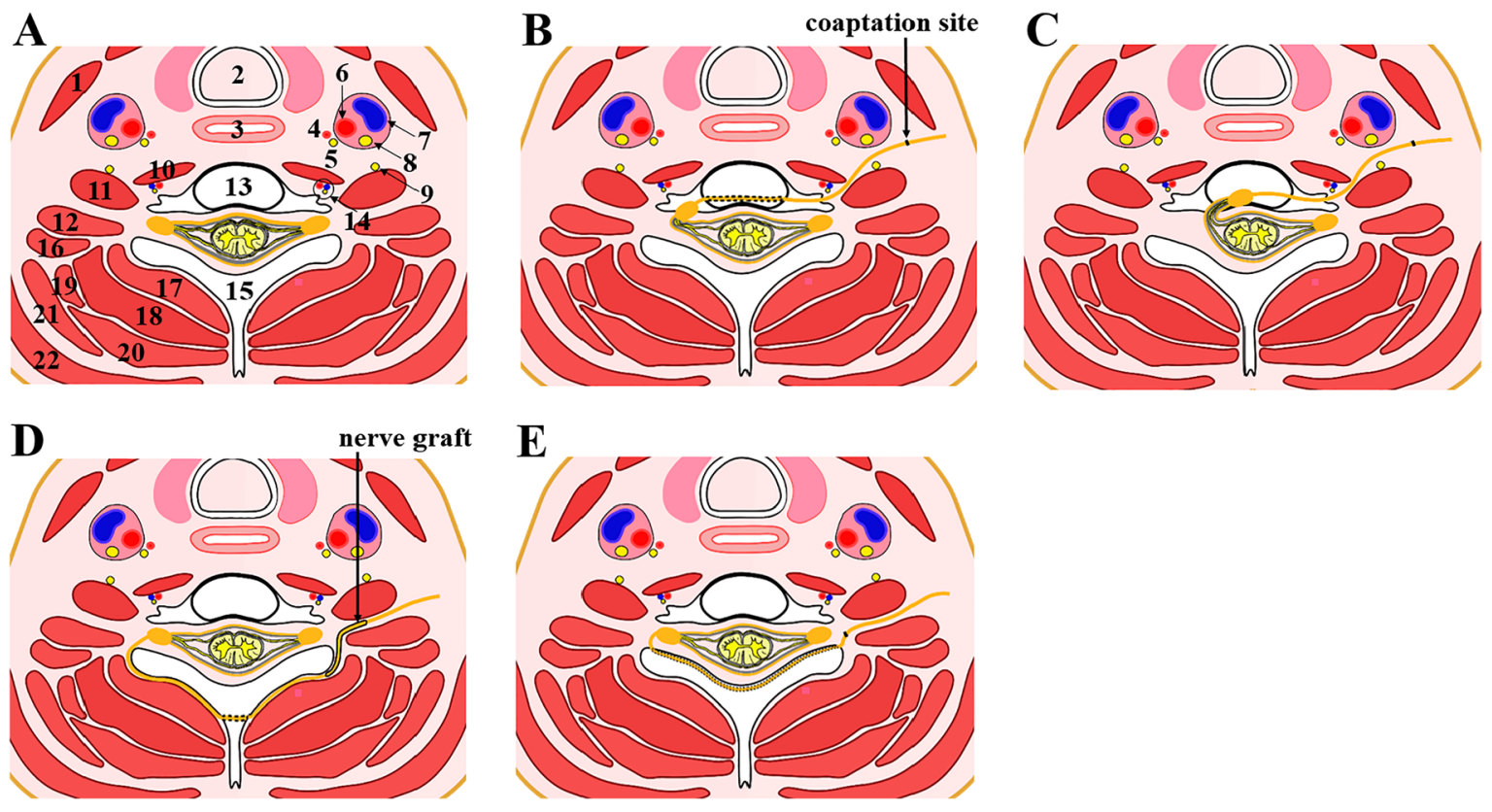

Figure 3: Schematic diagrams of vertebral routes and post-spinal routes. (A) A representative axial section through the $C 7$ vertebral body showing normal cervical anatomical structures. (B-E) Diagrams of the vertebral body channel route (B), the intervertebral disc channel route (C), the original post-spinal route (D), and the modified post-spinal route by Guan et al. (E). The anatomical structures marked by (1-15) are the same as those described in Figure 1; (16) posterior scalene muscle, (17) semispinalis cervicis muscle, (18) semispinalis capitis muscle, (19) splenius cervicis muscle, (20) splenius capitis muscle, (21) levator scapulae muscle, and (22) trapezius muscle. 
vertebrae is far greater than the diameter of the $\mathrm{C} 7$ nerve, allowing for the re-routing of the CC7. These anatomical features create the condition for the establishment of the posterior route. For surgical procedures, a supraclavicular transverse incision was performed for the dissection of the CC7. Then, the cadaver was placed in the prone position for the exposure of the vertebral laminae and spinous processes of the $\mathrm{C} 7$ and $\mathrm{T} 1$ via the cervical posterior approach. The interspinal ligament between the $\mathrm{C} 7$ and $\mathrm{T} 1$ was then drilled near the lamina to form a 6-mm diameter channel. The CC7 was diverted backwards circumventing the articular processes and transferred towards the opposite side via the hole. Post-operative passive movement of the neck, including flexion, extension, and lateral curvature, did not compress the transferred nerve. However, anatomical measurements showed that the distance between the proximal end of $\mathrm{C} 7$ and contralateral upper or lower trunk through the post-spinal route was significantly longer than that through the prespinal route $(7.21 \pm 1.02 \mathrm{~cm}$ vs. $5.61 \pm 1.26 \mathrm{~cm}$ for upper trunk; $9.52 \pm 1.25 \mathrm{~cm}$ vs. $7.17 \pm 1.59 \mathrm{~cm}$ for lower trunk), which was longer than the length of C7 nerve plus posterior $(6.51 \pm 0.91 \mathrm{~cm})$ or anterior division $(7.00 \pm 1.08 \mathrm{~cm})$ (Xiang et al. 2012). In addition, the authors also tried to re-route CC7 through the interspinous space of $\mathrm{C} 6$ and $\mathrm{C} 7$, but the distal end of the CC7 got past the midline only $1 \mathrm{~cm}$ (Li et al. 2012). Hence, this route with neurorrhaphy designed out of the vertebral canal has no advantage of shortening the nerve gap compared to the pre-spinal route.

\section{The modified post-spinal route}

Inspired by the posterior approach of cervical spine neurosurgery, we established a novel post-spinal route for CC7 transfer in the treatment of spastic hemiplegia in 2016 (Figure 3E) (Guan et al. 2019). On the basis of Xiang et al.'s procedure (Xiang et al. 2012), bilateral articular processes were resected to create a bone window. Then, the spinous ligament between the $\mathrm{C} 6$ and $\mathrm{C} 7$ as well as adjacent spinous processes was perforated to form a 1-cm diameter channel behind the ligament flavum. The resected distal end of the CC7 was withdrawn through the bone window and then rerouted across the channel to suture with the recipient $\mathrm{C} 7$. The advantage of this route is that opening the articular processes enables the transfer of the $\mathrm{CC} 7$ through the intervertebral foramen and further shortens transferring distance comparing to circumventing the articular processes posterolaterally, which makes direct tension-free anastomosis possible in all the 10 patients. Short-term follow-up results showed that more than half of the patients had lower spasticity and better sensation after operation (Guan et al.
2019, 2020), and a long-term follow-up was still in process for evaluating the restoration of motor function.

This approach, widely used in cervical discectomy decompression surgeries, is familiar to spine surgeons. One major limitation of this route is that the shift in operative position during surgery demands extra anesthesia management (Feng et al. 2019a). In addition, people are deeply concerned that the damage to facet joints greatly impairs the stability of cervical spine, which may cause nerve traction during neck movement and subsequently increase the risk of poor nerve regeneration and sensory disturbances (Feng et al. 2019a). Indeed, we totally agree with these disadvantages. During surgery, we tried to sacrifice fewer articular processes to ensure CC7 transfer, and maintain other important structures including the ligament flavum, the supraspinal ligament, and the rest of the interspinous ligament. Bone graft fusion or the fixation of lateral mass screws was also performed to increase the stability of the spine. As recommended by (Feng et al. 2019a), postoperative immobilization should also be longer and stricter. Considering these, avoiding the risk of cervical instability is urgently needed for improving this route.

Considering the impact of spasticity on functional recovery after CC7 transfer (Petuchowksi et al. 2018), we propose that the combination with SPN or SDR of the affected cervical nerve will further improve spasticity and promote rehabilitation. Recently, a combined surgery of SDR and CC7 transfer was implemented in one patient with diffuse upper-limb spasticity owing to cerebral hemorrhage in the basal ganglia in our center. Although immediate alleviation of spasticity was seen after surgery, a longer follow-up is required to fully determine the safety and efficacy of this technique (Guan et al. 2021). A prospective study with greater sample size is also necessitated to determine if this combination surgery exerts synergistic effect than CC7 transfer or SDR/SPN alone.

\section{The posterior intradural route}

In 2019, Jiang et al. firstly established a posterior intradural approach for treating hemiplegic upper-limbs in a cadaver feasibility study (Figure 4A, B). In this route, the spinous process and lamina of the $\mathrm{C} 6, \mathrm{C} 7$, and $\mathrm{T} 1$ were removed via cervical posterior approach, with the drilling of the facets restricted to about $30 \%$ of the width of the joint. Then, the dura was incised in the midline, and the arachnoid was separated to clearly expose both ventral roots (VR) and dorsal roots (DR) of $\mathrm{C} 6, \mathrm{C} 7$, and C8 bilaterally. After the transection of dentate ligaments, nerve roots in the healthy side were divided as distally as possible, while those in the 
injured side were divided as proximally as possible. In this case, precise neurorrhaphy was successfully performed on the surface of the dorsal spinal cord connecting DRs to DRs (with a 1-cm graft) and VRs to VRs (with a 2-cm graft) of bilateral $\mathrm{C} 7$. Multiple anastomoses of the $\mathrm{C} 7$ to various roots on the contralateral side were also possible within the same surgical field with the aid of interposition nerve grafts. The dura was closed after completion of neurorrhaphy. This kind of multiple anastomose enables full use of the donor $\mathrm{C} 7$ and precise repair (Jiang et al. 2019). However, several potential risks should be considered before clinical application. Firstly, the removal of vertebral structures would impair the structural integrity. Secondly, opening the dura mater may cause epidural hematoma, infection, cerebrospinal fluid leak, and spinal injury. Thirdly, neural anastomose may compress the spinal cord within the subarachnoid space. Fourthly, the anti-tension strength of the anastomosed nerves was weak since intradural nerve roots were only enwrapped by pia mater. Last, the separation or identification of the VRs and DRs may be difficult since they were divided into multiple bundles originating from the spinal cord (Jiang et al. 2019; Yang et al. 2020).

\section{The posterior extradural route}

In 2020, Yang et al. demonstrated the feasibility of extradural nerve anastomosis via cervical posterior approach for treating hemiplegic upper-limbs in a cadaver study (Figure 4C) (Yang et al. 2020). Differing from the intradural route (Jiang et al. 2019), the extradural VRs and DRs of bilateral C7 were separated after the visualization of the $\mathrm{C} 7$ dorsal root ganglion in the epidural space, taking care to protect the adjoining vertebral artery. The CC7 nerve root was cut off at the junction of the VR and DR, while the recipient $\mathrm{C} 7$ cut off at the nerve root outlet of the dura mater. In all the nine cadavers, the VR and DR of $\mathrm{CC} 7$ were directly anastomosed to those of the recipient $\mathrm{C} 7$ on the dura mater, respectively. Compared with the intradural route, longer nerve roots could be procured via the extradural route. In addition, these extradural nerve roots, enwrapped in dura mater, have higher anastomosis strength, which enables easier and more accurate identification of the VR and the DR. All these merits allow for direct, precise and tension-free coaptation of bilateral C7 nerves (Yang et al. 2020). Compared with current mixed-nerve anastomosis, precise anastomosis (motor-tomotor connection and sensory-to-sensory connection) will inevitably reduce misdirected nerve regeneration and then improve nerve recovery. The anastomosed nerves did not exert obvious compressive effect on the spinal cord evidenced by postoperative imaging data. For the concern of laminectomy, the fixation of lateral mass screws is recommended to strengthen spinal stability (Yang et al. 2020). However, a major problem of the intra-spinal routes is that pre-ganglionic sectioning was performed in the recipient side within the vertebral canal. This pre-ganglionic neurorhaphy may impede the axon regrowth toward the distal end. Thus, further studies are required to confirm its clinical feasibility.

\section{Brain involvement in functional recovery after $\mathrm{CC} 7$ transfer}

The continuous refinement of the surgical approach for CC7 transfer ultimately achieves direct anastomosis of the $\mathrm{CC} 7$ and the recipient nerves. However, only maximizing peripheral nerve regeneration is not enough to gain favorable clinical outcomes. As a procedure of rearranging the peripheral nerve pathway, CC7 transfer has been proven to induce adaptive cerebral remodeling (Jiang et al. 2010; Sun et al. 2014). Thus, efforts to improve the outcome should address the brain as well (Jiang et al. 2010; Sun et al. 2014).
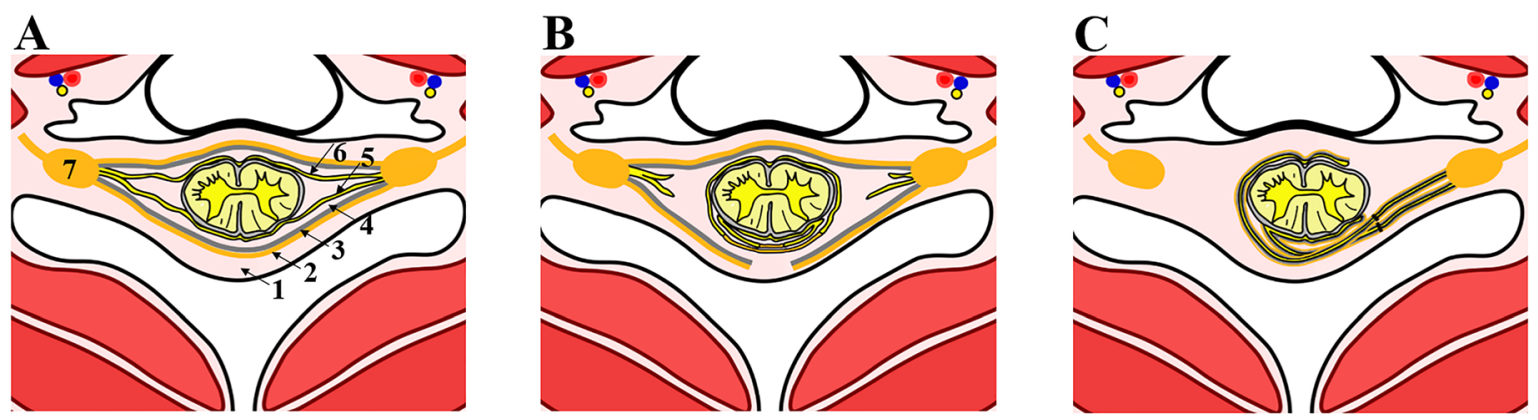

Figure 4: Schematic diagrams of intra-spinal routes. (A) A representative axial section through the $C 7$ vertebral body showing normal anatomical structures within the vertebral canal. (B and C): Diagrams of the posterior intradural route (B), and the posterior extradural route (C). (1) epidural space, (2) dura meter, (3) arachnoid, (4) subdural space, (5) dorsal roots, (6) ventral roots, and (7) dorsal root ganglion. 


\section{Transhemispheric organization of motor cortex after CC7 transfer for BPAI}

From the perspective of neuroanatomy, $\mathrm{CC} 7$ transfer connects the injured hand to the cerebral hemisphere ipsilateral to the damaged plexus, which mainly controls contralesional upper-extremity. Therefore, regaining independent motor and sensory function is a key indicator for evaluating the surgical efficacy in patients with BPAI. Indeed, synchronous motion and sensation of contralesional healthy upperextremity is a common phenomenon after CC7 transfer. In the early stage of reinnervation, a patient is able to coordinately move the paralyzed hand only with volitional movements of the healthy hand. Over several years, some patients gain active movements as the synchronous motion in the healthy side decreases or even disappears (Gu et al. 1992, 1998). A similar phenomenon was also seen in the rat model of CC7 transfer, which could be explained by a dynamic process of interhemispheric functional reorganization in the motor cortex (Figure 5B). Neurophysiological evidence showed that in rats with BPAI, the cortical representation area for the injured forelimb was mainly replaced by that of the neck. After nerve repair, the motor center for the paralyzed forepaw firstly emerged in the hemisphere ipsilateral to the injured side, where it shared great overlapping with that of the healthy forepaw. Then, the silent original motor center of the injured forelimb in the contralesional hemisphere regained activation, which initiated bilateral control of the injured limb. Finally, the contralesional motor center gained exclusive control of the injured forelimb (Jiang et al. 2010; Lou et al. 2006). Similar process was also seen in the brain of patients with CC7 transfer evidenced by functional imaging data (Beaulieu et al. 2006; Hua et al. 2013; Ma et al. 2018; Yu et al. 2017; Zuo et al. 2010).

Animal studies showed that transhemispheric functional reorganization was disrupted by corpus callosotomy in the early stage after CC7 transfer (Hua et al. 2012a). Imaging data further documented that the corpus callosum
A

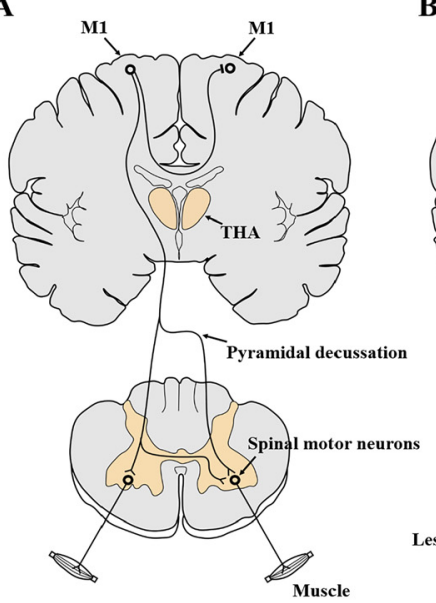

B

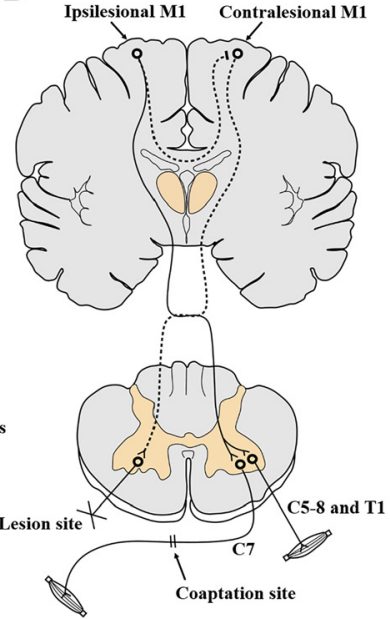

C

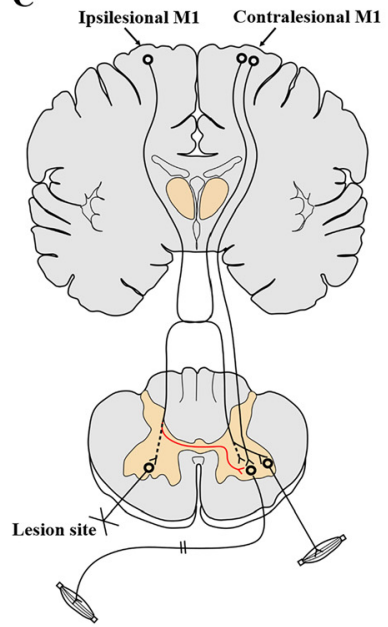

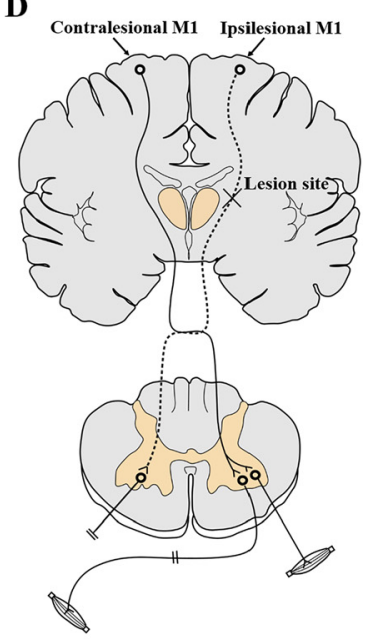

— Neural pathways exerting functions after surgery

$\longrightarrow$ Excitatory neural connections

- Recrossed cortical spinal tracts

Figure 5: Scheme depicting potential neural pathways involved in motor function recovery after CC7 transfer. (A) Schematic representations of corticospinal fibers originating from the $\mathrm{M} 1$ in one hemisphere (the left side). Most corticospinal fibers decussate in the medulla and at the targeted spinal level before synapsing with contralateral lower motor neurons, while the uncrossed part innervate ipsilateral lower motor neurons. In addition, there exists interhemispheric functional inhibition between bilateral M1 regions to ensure motor dexterity. (B) Potential pathways involved in the early phase of motor recovery after surgery in the treatment of BPAl. The ipsilesional arm is firstly innervated by ipsilesional M1 via the lateral CST originating from the ipsilesional side, which also controls the healthy limb, thus leading to synchronous motion. Simultaneously, the interhemispheric callosal inhibition between bilateral M1 weakens, which initiates transhemispheric motoric reorganization. (C) Potential pathways involved in the late phase of motor recovery after surgery in the treatment of BPAI. When transhemispheric motoric reorganization finishes, the contralesional M1 gradually gains control of the injured arm possibly via the anterior CST and the recrossed lateral CST from the contralesional side. This functional compensation leads to functional retraction of ipsilesional lateral CST toward the transferred CC7 via competition mechanisms, which ultimately enables contralesional M1 to gain exclusive control of the injury arm. (D) Potential pathways involved in motor recovery after surgery in the treatment of central hemiplegia. The ipsilesional M1 loses control of body movement owing to the devastating damage. Instead, the contralesional M1 exerts compensatory control of the injured arm via the lateral CST. BPAI, brachial plexus avulsion injury; CC7, contralateral C7 nerve; CST, corticospinal tracts; M1, primary motor cortex; THA, thalamus. 
was activated during the movement of the injured hand, which was highly correlated with motor recovery (Hua et al. 2012b; Li et al. 2015a; Ma et al. 2018). These available literature suggest, as an essential bridge of long-range plasticity following CC7 transfer, the corpus callosum may transmit sensory feedback signals from movements evoked by ipsilesional cortex to its contralesional counterpart. Under normal condition, transcallosal interhemispheric connections usually exert inhibitory effects on contralateral motor areas to inhibit mirror movements, which is imperative for motor dexterity (Allison et al. 2000). However, this interhemispheric inhibition is blocked after dramatic alterations of the peripheral pathway, such as BPAI and CC7 transfer (Hsieh et al. 2002; Hua et al. 2012b). The resultant disinhibition of contralesional motor cortex is deemed to initiate the functional control over the paralyzed extremity (Figure 5B) (Lou et al. 2006).

The mechanisms underlying the formation of functional neurocircuitry between contralesional primary motor cortex (M1) and the transferred CC7 nerve have not been visited yet. The first possibility is that contralesional M1 activates ipsilesional M1 via interhemispheric connections and then controls the transferred CC7. However, results of animal experiments showed ablation of ipsilesional M1 in the late phase of transhemispheric motor cortex reorganization failed to prevent the contralesional cortex from evoking movements in the injured forepaw (Lou et al. 2006). Therefore, corticofugal projections from the contralesional hemisphere may subserve the control over the transferred CC7. As we know, approximately $90 \%$ of the fibers in the CST decussate in the medulla to form the lateral CST, while the rest continue descending within ipsilateral spinal cord, known as the anterior CST, and decussates in the spinal cord or not before synapsing with lower motor neurons (Figure 5A) (Canedo 1997). Hence, the anterior CST may participate in the control of the transferred CC7. In addition, indirect actions via descending reticulospinal or vestibulospinal tracts and segmental interneurons in the contralesional side may also be involved in this process (Jankowska and Edgley 2006). Another potential mechanism is the rewiring of lateral CST originating from the contralesional hemisphere, which recrosses spinal midline to reach the motoneurons of the transferred CC7, which is usually seen under the condition of unilateral lesion of motor cortex in both neonatal rodents and patients (Barth and Stanfield 1990; Carr et al. 1993; Jankowska and Edgley 2006; Kuang and Kalil 1990). It is possible that the newly-formed synapses of contralesional CST with CC7 motoneurons further lead to gradual functional withdrawal of ipsilesional CST axons via competition (Lou et al. 2006). These hypotheses remain to be confirmed in the future (Figure $5 \mathrm{C}$ ).

Apart from M1, the premotor cortex, supplementary motor cortex, and posterior parietal areas were also activated during motor imagery and motor execution after $\mathrm{CC} 7$ transfer (Beaulieu et al. 2006; Wang et al. 2015). The activation of premotor cortex and precuneus was proven to correlate with motor recovery in the central remolding course after CC7 transfer (Ma et al. 2018). Among these regions, supplementary motor cortex and premotor cortex are involved in the planning and execution of movements, the attainment of motor skills, as well as the selection of appropriate motions through coordination with other areas (Deiber et al. 1998; Vorobiev et al. 1998), while premotor cortex and precuneus may coordinate in the process of visual guidance of limb movements (Hayashi and Yokoi 2016; Wise et al. 1997). Hence, the activation of these high-order motor areas may facilitate the relearning process and eventually benefits motor rehabilitation after CC7 transfer. Considering these, rehabilitation training (e.g. motor imagery) for recruiting high-order motor areas (Bovend'Eerdt et al. 2010) may improve functional recovery after $\mathrm{CC} 7$ transfer.

\section{Intrahemispheric organization of sensory cortex after CC7 transfer for BPAI}

For sensory recovery, touching the injured hand induces a sensation of tingling only in the healthy hand in the early phase of regeneration, and then in bilateral hands as time progresses (Chen et al. 2007; Lanaras et al. 2009). As mentioned above, patients could gain independent movement of the injured extremity as synchronous motion decreases. However, the sensory perception of the injured hand could not be separated from the healthy hand over time (Chen et al. 2007; Lanaras et al. 2009). Recent animal studies showed that unlike transhemispheric organization of motor cortex after CC7 transfer, the sensory function of the injured hand was dominated by ipsilesional primary sensory cortex (S1) (Wang et al. 2010). A great overlapping of sensory representation areas was seen between the injured hand and the intact hand, the ratio of which was significantly related with the synchronous sensation (Cai et al. 2021; Wang et al. 2010), suggesting the aberrant S1 plasticity in might account for the abnormal sensation after CC7 transfer. A possible hypothesis for this aberrant plasticity is the lack of direct connection between homotopic sensory regions of hand representation areas. Anatomical evidence showed that the proximal and midline body part representations in the S1 are densely interconnected via the callosum, whereas the representations of extremities 
are sparsely interconnected or even separated (Jones and Peters 1986). Thus, the brain fails to initiate transhemispheric functional reorganization of $\mathrm{S} 1$ after CC7 transfer.

Sensory-motor integration is fundamental for motor reconstruction (Wolpert and Flanagan 2010). However, the synchronous sensation induces misperception of the injured hand, which potentially influences motor learning of the injured hand by impeding normal sensory feedback circuits. Thus, restoring sensory function of the paralyzed hand is important for the overall recovery (Bolognini et al. 2016). Two major issues remain to be investigated in the future: how to separate the overlapping areas of the two hands and how to reactivate the silent contralesional S1 of the injured hand. Central interventional methods, including sensory cortex stimulation, visual-sensory feedback rehabilitation, and tactile enhancement methods may hold promise for solving this problem (Cai et al. 2021).

\section{Peripheral factors affecting cortical reorganization after CC7 transfer for BPAI}

One interesting phenomenon is that cortical reshaping pattern after CC7 transfer is usually influenced by peripheral factors, including the mode of $\mathrm{CC} 7$ nerve division, the mode of nerve reconstruction, as well as the type of recipient muscle. This knowledge will not only deepen our understanding about the action mode of cortical remodeling after $\mathrm{CC} 7$ transfer, but also help optimizing this surgery to attain a favorable outcome.

As mentioned before, the efficacy of using entire $\mathrm{CC} 7$ as the donor is superior to partial root sectioning (Hierner and Berger 2007; Sammer et al. 2012; Tu et al. 2014). Besides the number of nerve fibers provided, cortical plasticity mechanism is also involved. In a recent study, rats with BPAI were treated with $\mathrm{CC} 7$ transfer to repair the median nerve with different division modes, including entire transection and transfer, entire transection but partial transfer, and partial transection and transfer. The authors found that for the same recipient nerve, harvesting of entire $\mathrm{CC} 7$ yielded better functional recovery than partial harvesting, even if only part of the entire root utilized for transfer. Correspondingly, harvesting of entire CC7 induced higher expression of brain derived neurotrophic factor (BDNF), a cortical reorganization biomarker, in bilateral M1 than partial transection, while no significant difference in BDNF levels was noted between entire transection and transfer group and entire transection but partial transfer group. These data suggest different divisions of $\mathrm{CC} 7$ nerve may result in different surgical effects through the modulation of cortical reorganization (Gao et al. 2018; Guo et al. 2021).
However, less is known about the BDNF levels in the spinal motor neurons as well as their distal axons, which remains to be explored in the future.

Apart from the division mode, the mode of nerve reconstruction also affects cerebral remodeling. Animal studies showed that $\mathrm{CC} 7$ transfer to the upper trunk or to both the musculocutaneous nerve and the median nerve made faster trans-hemispheric motoric reorganization than that to the median nerve alone in rats (Pan et al. 2012). This phenomenon is correlated with the fact that the former reconstruction mode markedly reduced the occurrence of synchronous movement compared to that to the median nerve alone (Chen et al. 2007). One possible explanation for this phenomenon is that reinnervation of muscles in the upper arm, especially the biceps, helps patients to achieve routine movements using the arm, which is difficult to achieve by the recovery of finger flexion alone. The enhanced rehabilitation training in turn promotes transhemispheric organization of motor cortex after CC7 transfer as well as regeneration from $\mathrm{CC} 7$ toward the injured side along the nerve graft (Pan et al. 2012).

Cortical remodeling also differs in restoring different motor functions following $\mathrm{CC} 7$ transfer. The recovery of intrinsic hand muscles for fine motor function is poor under the condition of brachial injury (Kosiyatrakul et al. 2009; Post et al. 2012) and even after CC7 transfer (Gu et al. 1992; Wang et al. 2011; Yang et al. 2018), and both peripheral and central factors are involved in this phenomenon. For irreversible atrophy of the intrinsic muscles (Carraro et al. 2015; Midrio 2006), it was originally attributed to the long instance between the damaged nerve and intrinsic muscles. However, a long-term follow-up study showed that even with transfer to the same recipient (the median nerve), the return of function in extrinsic hand muscles (e.g., the flexor digitorum) was better than that in intrinsic hand muscles (e.g., the abductor pollicis brevis) if successful reinnervation was accomplished. This discrepancy suggests regeneration distance might not be the mere attribution to unfavorable outcomes of intrinsic muscles (Li et al. 2015a; Wang et al. 2011). Interestingly, it was reported that expression profiles of both miRNAs and mRNAs in forepaw intrinsic muscles were different from those of the reversible biceps in the rat model of obstetric BPI. Thus, lack of self-repair potential in neuromuscular junctions was proposed as a major reason why atrophy intrinsic muscles became irreversible after denervation (Pan et al. 2015; Wu et al. 2016). Apart from peripheral causes, emerging imaging evidence showed that the cortical representation of flexor digitorum was located in bilateral hemispheres, whereas that of abductor pollicis brevis only in the ipsilesional cortex after CC7 transfer. Then, the 
authors realized that the absence of interhemispheric plasticity may also be responsible for the poor recovery of intrinsic muscles (Li et al. 2015a). Considering these, therapeutics aiming at restoring transhemispheric reorganization of motor cortex corresponding to intrinsic muscles (e.g., motor cortex stimulation) may be conducive to the gain of fine hand function after $\mathrm{CC} 7$ transfer.

\section{Cortical reorganization after $\mathrm{CC} 7$ transfer for central hemiplegia}

Contralesional hemispheric compensation is an important recovery mechanism of upper-extremity after severe unilateral brain lesion (Lotze et al. 2006; Netz et al. 1997). However, this form of compensation is relatively limited owing to scarce projections via uncrossed CST from contralesional hemisphere to the paralyzed extremity (Hua et al. 2016). Hence, CC7 transfer is needed to enhance this neural connection via crossed CST. Imaging evidence showed that in patients with brain injury who did not receive $\mathrm{CC} 7$ transfer, the weak activation in the ipsilesional hemisphere generated by voluntary movement of the paralyzed extremity remained unchanged during a 12-month period. However, in those receiving CC7 transfer, the pre-existing activation in the ipsilesional hemisphere decreased at month 12 comparing to that of baseline, and with it comes the activation of the contralesional hemisphere which emerged at postoperative month 8 and continued to increase until month 12 (Zheng et al. 2018). A similar process was seen in rodent brain injury model with CC7 transfer (Hua et al. 2016). These data suggest that the residual ipsilesional hemisphere contributes to spontaneous recovery after brain injury, while the newly-formed adaptive plasticity in the contralesional hemisphere contributes to the significant motor recovery of the paralyzed forelimb after CC7 transfer (Hua et al. 2016) (Figure 5D).

\section{Motor cortex stimulation for boosting functional recovery after $\mathrm{CC} 7$ transfer}

Above evidence regarding M1 in functional recovery of CC7 transfer imply that M1 may hold promise as a potential rehabilitation therapeutic target. As the most rostral part of the motor pathway, M1 is easily accessible by electrical stimulation, which was firstly introduced into the field of neuropathic pain therapy. Neuropathic pain is the pain caused by a lesion in or disease of the somatosensory nervous system (Treede et al. 2008). Brachial plexus injury would lead to peripheral neuropathic pain in more than $50 \%$ patients (Ciaramitaro et al. 2017; Tantigate et al. 2015), while stroke is the leading cause resulting in central neuropathic pain among various types of central neurological injury, with a reported incidence of 1-8\% in patients with stroke (Andersen et al. 1995; Bowsher 1993; Ciaramitaro et al. 2017; Singer et al. 2017). Central post-stroke pain (CPSP) always develops within the area of sensory disturbances, and is described as burning, numb, aching, or pricking. Although the pathophysiology still remains elusive, it is considered as a disorder of brain network remodeling, which is characterized by thalamic hyperexcitability (Hosomi et al. 2015). This refractory pain often interferes post-stroke rehabilitation, thereby reducing individual quality of life (Andersen et al. 1995; Bowsher 1993). Based on experimental observations that stimulating cortical or subcortical motor fibers suppressed noxious signal transmission within the spinal dorsal horn (SDH) (Coulter et al. 1974; Lindblom and Ottosson 1957), MCS was firstly introduced by (Tsubokawa et al. 1991, 1993) as a treatment for CPSP, and later proved efficacious for other types of neuropathic pain (Brown and Barbaro 2003; Nguyen et al. 2011). The stimulation paradigm Tsubokawa et al. used was to surgically implant plate electrodes in the epidural space over motoric areas topographically related to the affected limb. Later, noninvasive paradigms, including repetitive transcranial magnetic stimulation (rTMS) and transcranial direct current stimulation (tDCS), gradually gained in popularity owing to its simplicity and safety (Fregni et al. 2006; Lefaucheur et al. 2001). Generally, MCS enhances the output activity of M1, by which modulates the activity of widespread cortical and deeper brain structures. For neuropathic pain control, MCS inhibits spinal pain processing via the descending pain modulatory system and reduces thalamic hyperactivity, thus influencing interwoven cortico-subcortical pain matrix which cover the sensory, affective and cognitive aspects of pain processing (Hosomi et al. 2015; Lefaucheur 2016).

In the domain of brain or spinal cord injury, strengthening spared connections from M1 has emerged as an important strategy for motor system repair (Hagg 2006; Jankowska and Edgley 2006). Thus, MCS was also adopted for enhancing CST axons sprouting after unilateral CST injury (Brus-Ramer et al. 2007), and has been proven to promote motor recovery both in rodent models and patients with stroke (Carmel et al. 2010; Kang et al. 2016; Lefebvre and Liew 2017). The mechanisms underlying MCS-related motor function recovery have been elucidated in experimental animal studies. As mentioned before, the contralesional CST, though sparse, has properties in restoring function after severe unilateral CST injury (Jankowska and Edgley 2006). Contralesional MCS has been documented to drive CST outgrowth to both sides of the spinal cord in a unilateral CST 
lesion rat model, but this outgrowth was stronger to the impaired side of the animal, which was denervated by the injury (Carmel et al. 2010). Coordinated outgrowth in the red nucleus may also contribute to the reparative effects via descending motoric modulation (Carmel et al. 2013). It is worth mentioning that MCS also has beneficial effects after peripheral nerve injury and repair in terms of nerve regeneration and muscle re-inervation in a rodent model (Nicolas et al. 2018). In view of above-mentioned merits in both motor recovery and pain control, we believe MCS may hold promise for boosting functional recovery after $\mathrm{CC} 7$ transfer.

The electrode montage of MCS is a key question in the treatment of central hemiplegia, which has implications for its application in functional recovery after $\mathrm{CC} 7$ transfer. Herein, M1-tDCS was taken for example to explain this issue. TDCS delivers low-intensity electric current through the skull to selected brain areas, which induces neural excitability changes via subthreshold modulation of resting membrane potential in a polarity-dependent way. Anodal tDCS enhances, while cathodal tDCS diminishes excitability, within certain stimulation duration and strength (Knotkova et al. 2013). The specific tDCS montage with analgesic effects is anodal stimulation delivered over M1, typically with the anode positioned over M1 contralateral to the painful side and the cathode over ipsilateral supraorbital region. However, tDCS electrode configuration for stroke motor recovery is complicated by motoric structure reserve (i.e., the quantity of motoric pathways spared by the lesion) (Kang et al. 2016). In the case of high structure reserve, the preserved ipsilesional CST is able to revive locomotion of contralesional paretic
A

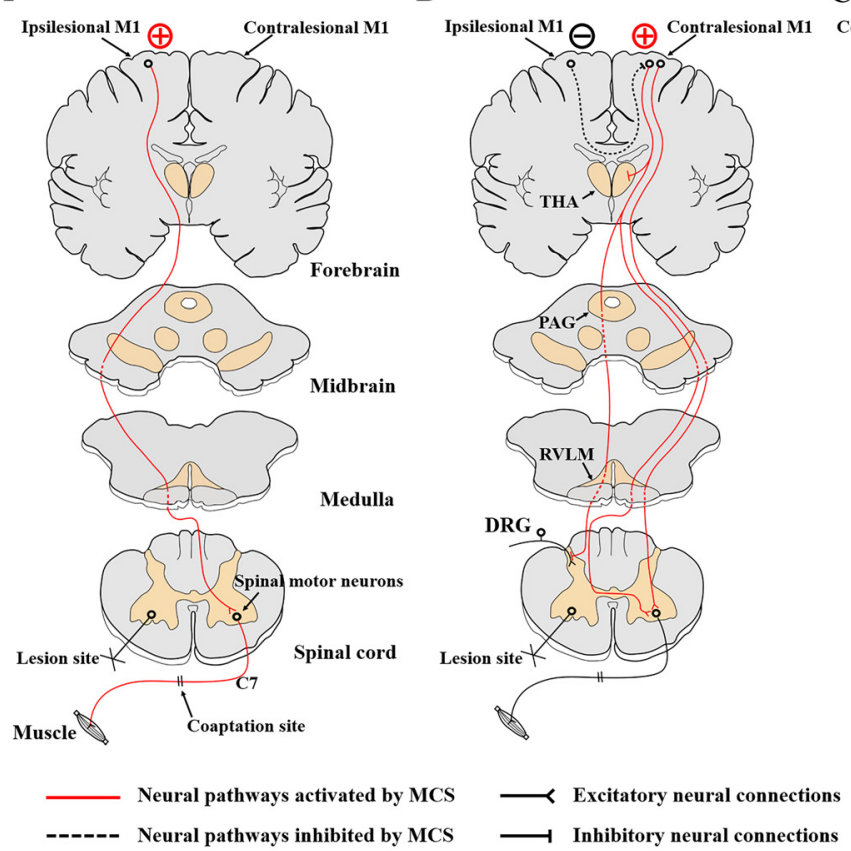

C

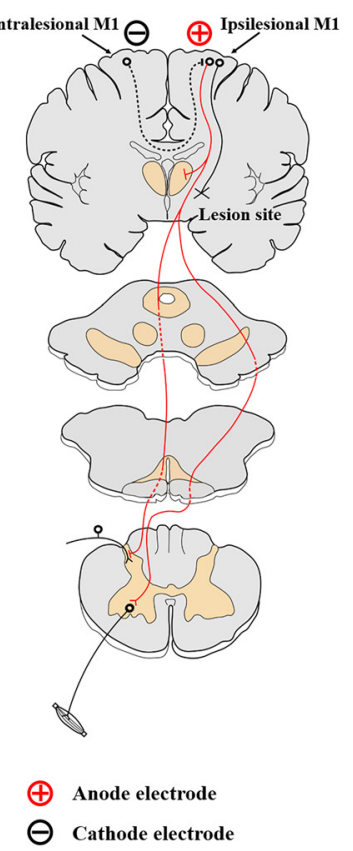

D

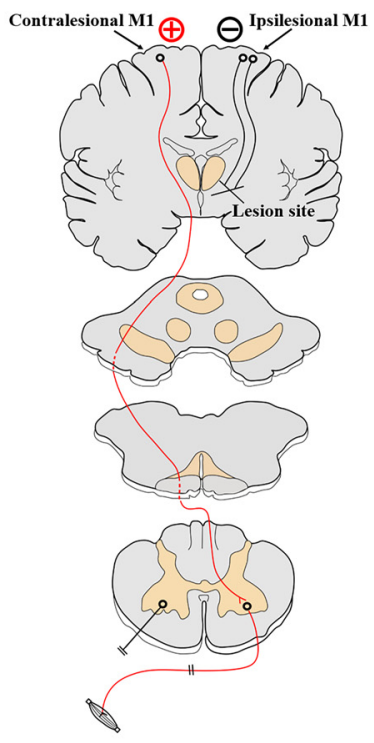

Figure 6: Scheme depicting MCS configuration and the underlying neurocircuity mechanisms in the treatment of upper extremity paralysis. (A) MCS configuration after CC7 transfer in the treatment of BPAI in the early phase. Anodal stimulation upon ipsilesional M1 could escalate its functional control over spinal motor neurons innervating the transferred $\mathrm{CC} 7$ as well as promote peripheral nerve regeneration after $\mathrm{CC} 7$ transfer. The cathode is placed in the contralesional supraorbital area, which is not shown in this figure. (B) MCS configuration after CC7 transfers in the treatment of BPAI in the later phase. The configuration with the cathodal upon ipsilesional M1 and the anodal on contralesional M1 reverses the interhemispheric inhibition from ipsilesional $M 1$ and facilitates transhemispheric motoric reorganization. The anodal subsequently boosts the outgrowth of the CST to connect with spinal motor neurons innervating the CC7 nerve. In addition, contralesional M1 activation also exerts analgesic effects through inhibiting the hyperactive thalamic nucleus. The descending pain inhibitory pathway, i.e. the PAG-RVLM-SDH pathway, is also recruited to modulate pain processing within the SDH. (C) MCS configuration in the treatment of central hemiplegia with high structure reserve. The reserved $\mathrm{M} 1$ is able to control the contralesional limb after functional compensation, so CC7 transfer is not necessitated. The anodal on ipsilesional M1 could boost the outgrowth of the CST, as well as lessen the neuropathic pain via pathways described before. (D) MCS configuration in the treatment of central hemiplegia with low structure reserve. The reserved $\mathrm{M} 1$ is unable to control the contralesional limb, thus CC7 transfer is needed to bridge the contralesional M1 and the injured limb. The anodal on contralesional M1 may enhance its functional connections toward spinal motor neurons innervating the transferred $\mathrm{CC} 7$ as well as peripheral nerve regeneration after CC7 transfer. BPAI, brachial plexus avulsion injury; CC7, contralateral C7 nerve; CST, corticospinal tracts; DRG, dorsal root ganglion; M1, primary motor cortex; MCS, motor cortex stimulation; PAG periaqueductal gray; RVLM, rostral ventrolateral medulla; SDH, spinal dorsal horn; THA, thalamus. 
extremity. However, inhibitory signals from the contralesional hemisphere may suppress the activity of M1 in the lesioned hemisphere, and therefore motor functional recovery and pain suppression mechanisms exerted by M1 may be malfunctioning (Morishita and Inoue 2016; Murase et al. 2004; Nowak et al. 2009). Hence, tDCS protocols aiming at balancing both excitatory and inhibitory activation between hemispheres, i.e. suppressing contralesional brain activity and/or enhancing ipsilesional activity, are recommended, include anodal stimulation on ipsilesional M1, cathodal stimulation on contralesional M1, or bilateral stimulation with the anodal on ipsilesional M1 and the cathodal on contralesional M1 (Kang et al. 2016). This bilateral stimulation paradigm has been adopted to treat CPSP recently (Morishita et al. 2015; Morishita and Inoue 2016) (Figure 6C). However, in the condition of low structure reserve, motor recovery of the paretic arm depends on functional compensation of contralesional hemisphere (Hummel et al. 2008; Jaillard et al. 2005). Thus, tDCS should aim at enhancing contralesional activity and/or suppressing ipsilesional hyperactivation (Hummel et al. 2008; Wu et al. 2013), which is contrary to that in the case of higher structure reserve. Considering that CC7 transfer is necessitated in the case of lower structure reserve after stroke, we speculate that M1-tDCS protocols should center on enhancing contralesional brain activity. MCS triggered lateral CST outgrowth originating from contralesional M1 to ipsilesional spinal cord (Carmel et al. 2010, 2013) will facilitate functional recovery of the paretic limb via the control of the transferred CC7 (Figure 6D).

Under the condition of BPAI, the stimulation paradigm may change with the phase of recovery after surgery. In the early phase, excitability-enhancing tDCS should be delivered over ipsilesional M1, with the aim to boost functional control of ipsilesional M1 over the transferred CC7 (Figure 6A). In the later phase when transhemispheric functional reorganization occurs, excitability-enhancing tDCS should be delivered over contralesional M1, typically with the anodal over contralesional M1 and the cathodal on ipsilesional M1. This montage shift could facilitate transhemispheric M1 organization and ultimately enable contralesional M1 to gain exclusive control of the injured arm (Figure 6B).

Another merit for the application of MCS after CC7 transfer is the analgesic effects. It is reported that MCS induces bilateral diffuse analgesic effects, although contralateral effects may be slightly higher (Nahmias et al. 2009; Passard et al. 2007). Thus, MCS contralateral to the side with pain is routinely recommended for pain control (Tsubokawa et al. 1991, 1993). In the condition of BPAI, the anode electrode is over contralesional M1, which exactly alleviate neuropathic pain induced by BPI. However, for the treatment of central hemiplegia, the anode electrode is located over the intact M1, the same side with CPSP. Whether contralesional MCS alleviates central pain remains to be investigated in the future.

\section{Conclusions and perspectives}

Establishing the shortest, safe, and effective route for CC7 transfer is the major concern of nerve repair doctors. Since 1986, the original approach has been constantly refined and innovated through decades of efforts of surgeons. In this review, we clearly observed an evolutional process of the surgical route from the superficial to the deep, and from front to back. Correspondingly, the choice of recipient nerves changed in order to restore the function of different target muscles and accommodate for various surgical routes in different diseases. The ultimate goal of these changes is to increase the efficiency of nerve regeneration via shortening the regeneration distance or even achieving direct coaptation.

We should dialectically recognize the values and limitations of each route. An individualized transfer route should be determined according to the condition of injury and individual anatomical variation, instead of blindly pursuing the shortest way. For the treatment of BPAI, the modified subcutaneous route and pre-spinal route could be considered according to the traumatic condition and the recipient nerve. For patients with central spastic hemiplegia, Huashan pre-spinal route remains as the standard approach yet. The post-spinal route established in our center also achieves direct anastomosis with proven clinical feasibility, highlighting the unique advantage of neuro-spinal surgery in the domain of nerve repair. However, further endeavor are needed to improve this route. Overall, it still needs a long time for clinical observation and evaluation of the clinical effects of CC7 transfer in the treatment of central hemiplegia. The vertebral route and the intra-spinal route are still in basic research phase, and there is a pressing need for the validation of clinical feasibility. It is also worth mentioning that high-resolution imaging techniques have advantages for structural imaging of the brachial plexus and the measurement of the length of $\mathrm{C} 7$, which has guiding significance for determining the surgical route $\mathrm{Yu}$ et al. 2019). Additionally, the introduction of minimally invasive techniques, including endoscopy and surgical robotassisted CC7 transfer, might also reduce surgical trauma (Jiang et al. 2016; Leblebicioglu et al. 2016).

Along with the progress of the surgical approach which maximizes peripheral nerve regeneration, central mechanisms should not be overlooked. Now we have gained a preliminary understanding of cerebral functional reorganization after $\mathrm{CC} 7$ transfer. However, the neurocircuitry basis 
for cerebral control of the paralytic arm is still unknown. The previous decade has witnessed the blossom of optogenetics in neuroscience, especially in the domain of motor control (Wang et al. 2017). The power of this tool lies in the ability to achieve regional and cell-type specific activation of neurocircuitry (Deisseroth 2015; Liu et al. 2020). Given favorable circumstances that this era presents, we should direct our focus toward a state-of-the-art integration of morphological, electrophysiological, behavioral, and optogenetic approaches to interrogate neurocircuitry mechanisms subserving motoric control after CC7 transfer. In addition, central intervening therapeutics, such as MCS, may also represent a remarkable advance in boosting functional recovery after CC7 transfer. Prospective studies will help to clarify the full benefit of this technique.

Author contributions: Y.B. and S.H. wrote the manuscript, drafted the figures and contributed equally to this work. J.G. and J.L. revised the manuscript. G.L. and M.Z. developed the concept and supervised the review.

Research funding: This work was supported by the National Natural Science Foundation of China (grant no. 81971133 to G.L. and 82101318 to Y.B.).

Conflicting of interest statement: No conflicts of interest are declared by the authors.

\section{References}

Aisen, M.L., Kerkovich, D., Mast, J., Mulroy, S., Wren, T.A., Kay, R.M., and Rethlefsen, S.A. (2011). Cerebral palsy: clinical care and neurological rehabilitation. Lancet Neurol. 10: 844-852.

Alawieh, A.M., Au Yong, N., and Boulis, N.M. (2021). Contralateral C7 nerve transfer for stroke recovery: new Frontier for peripheral nerve surgery. J. Clin. Med. 10, https://doi.org/10.3390/ jcm10153344.

Allison, J.D., Meador, K.J., Loring, D.W., Figueroa, R.E., and Wright, J.C. (2000). Functional MRI cerebral activation and deactivation during finger movement. Neurology 54: 135-142.

Andersen, G., Vestergaard, K., Ingeman-Nielsen, M., and Jensen, T.S. (1995). Incidence of central post-stroke pain. Pain 61: 187-193.

Aquilina, K., Graham, D., and Wimalasundera, N. (2015). Selective dorsal rhizotomy: an old treatment re-emerging. Arch. Dis. Child. 100: 798-802.

Barth, T.M. and Stanfield, B.B. (1990). The recovery of forelimbplacing behavior in rats with neonatal unilateral cortical damage involves the remaining hemisphere. J. Neurosci. 10: 3449-3459.

Beaulieu, J.Y., Blustajn, J., Teboul, F., Baud, P., De Schonen, S., Thiebaud, J.B., and Oberlin, C. (2006). Cerebral plasticity in crossed $\mathrm{C} 7$ grafts of the brachial plexus: an fMRI study. Microsurgery 26: 303-310.

Berman, J.S., Birch, R., and Anand, P. (1998). Pain following human brachial plexus injury with spinal cord root avulsion and the effect of surgery. Pain 75: 199-207.
Bertelli, J.A., Taleb, M., Mira, J.C., and Ghizoni, M.F. (2005). Variation in nerve autograft length increases fibre misdirection and decreases pruning effectiveness: an experimental study in the rat median nerve. Neurol. Res. 27: 657-665.

Bolognini, N., Russo, C., and Edwards, D.J. (2016). The sensory side of post-stroke motor rehabilitation. Restor. Neurol. Neurosci. 34: 571-586.

Bovend'Eerdt, T.J., Dawes, H., Sackley, C., Izadi, H., and Wade, D.T. (2010). An integrated motor imagery program to improve functional task performance in neurorehabilitation: a singleblind randomized controlled trial. Arch. Phys. Med. Rehabil. 91: 939-946.

Bowsher, D. (1993). Sensory consequences of stroke. Lancet 341: 156. Brown, J.A. and Barbaro, N.M. (2003). Motor cortex stimulation for central and neuropathic pain: current status. Pain 104: 431-435.

Brus-Ramer, M., Carmel, J.B., Chakrabarty, S., and Martin, J.H. (2007). Electrical stimulation of spared corticospinal axons augments connections with ipsilateral spinal motor circuits after injury. J. Neurosci. 27: 13793-13801.

Cai, Z., Lei, G., Li, J., Shen, Y., Gu, Y., and Feng, J. (2021). Aberrant central plasticity underlying synchronous sensory phenomena in brachial plexus injuries after contralateral cervical seventh nerve transfer. Brain Behav. 11: e02064.

Canedo, A. (1997). Primary motor cortex influences on the descending and ascending systems. Prog. Neurobiol. 51: 287-335.

Carmel, J.B., Berrol, L.J., Brus-Ramer, M., and Martin, J.H. (2010). Chronic electrical stimulation of the intact corticospinal system after unilateral injury restores skilled locomotor control and promotes spinal axon outgrowth. J. Neurosci. 30: 10918-10926.

Carmel, J.B., Kimura, H., Berrol, L.J., and Martin, J.H. (2013). Motor cortex electrical stimulation promotes axon outgrowth to brain stem and spinal targets that control the forelimb impaired by unilateral corticospinal injury. Eur. J. Neurosci. 37: 1090-1102.

Carr, L.J., Harrison, L.M., Evans, A.L., and Stephens, J.A. (1993). Patterns of central motor reorganization in hemiplegic cerebral palsy. Brain 116: 1223-1247.

Carraro, U., Boncompagni, S., Gobbo, V., Rossini, K., Zampieri, S., Mosole, S., Ravara, B., Nori, A., Stramare, R., Ambrosio, F., et al. (2015). Persistent muscle fiber regeneration in long term denervation. Past, present, future. Eur. J. Transl. Myol. 25: 4832.

Chen, L. and Gu, Y.D. (2001). Lowest number of brachial plexus nerve roots required for maintaining normal limb function-an experimental study. Hand Surg. 6: 37-45.

Chen, L., Gu, Y.D., Hu, S.N., Xu, J.G., Xu, L., and Fu, Y. (2007). Contralateral C 7 transfer for the treatment of brachial plexus root avulsions in children - a report of 12 cases. J. Hand Surg. Am. 32: 96-103.

Chuang, D.C., Cheng, S.L., Wei, F.C., Wu, C.L., and Ho, Y.S. (1998). Clinical evaluation of $C 7$ spinal nerve transection: 21 patients with at least 2 years' follow-up. Br. J. Plast. Surg. 51: 285-290.

Ciaramitaro, P., Padua, L., Devigili, G., Rota, E., Tamburin, S., Eleopra, R., Cruccu, G., and Truini, A. (2017). Prevalence of neuropathic pain in patients with traumatic brachial plexus injury: a multicenter prospective hospital-based study. Pain Med. 18: 2428-2432.

Coulter, J.D., Maunz, R.A., and Willis, W.D. (1974). Effects of stimulation of sensorimotor cortex on primate spinothalamic neurons. Brain Res. 65: 351-356. 
Deiber, M.P., Ibañez, V., Honda, M., Sadato, N., Raman, R., and Hallett, M. (1998). Cerebral processes related to visuomotor imagery and generation of simple finger movements studied with positron emission tomography. Neuroimage 7: 73-85.

Deisseroth, K. (2015). Optogenetics: 10 years of microbial opsins in neuroscience. Nat. Neurosci. 18: 1213-1225.

Dobkin, B.H. (2005). Clinical practice. Rehabilitation after stroke. N. Engl. J. Med. 352: 1677-1684.

Doshi, P.B. and Bhatt, Y.C. (2016). Passage through the carotid sheath: an alternative path to the pre-spinal route for direct repair of contralateral $\mathrm{C} 7$ to the lower trunk in total brachial plexus root avulsion injury. Indian J. Plast. Surg. 49: 159-163.

Enslin, J.M.N., Langerak, N.G., and Fieggen, A.G. (2019). The evolution of selective dorsal rhizotomy for the management of spasticity. Neurotherapeutics 16: 3-8.

Feng, J., Qiu, Y., Jiang, S., Xu, J., Yin, H., Li, T., Lei, G., Cai, Z., Shen, J., $\mathrm{Yu}$, B., et al. (2019a). Letter to the Editor regarding "treatment of central paralysis of upper extremity using contralateral C7 nerve transfer via posterior spinal route". World Neurosurg. 128: 618-619.

Feng, J., Wang, T., Gu, Y., Chen, L., Zhang, G., and Zhu, Y. (2010). Contralateral C7 transfer to lower trunk via a subcutaneous tunnel across the anterior surface of chest and neck for total root avulsion of the brachial plexus: a preliminary report. Neurosurgery 66: 252-263, discussion 263.

Feng, J., Wang, T., and Luo, P. (2019b). Contralateral C7 transfer to lower trunk via a subcutaneous tunnel across the anterior surface of the chest and neck for total brachial plexus root avulsion: a cadaveric study. J. Orthop. Surg. Res. 14: 27.

Fregni, F., Boggio, P.S., Lima, M.C., Ferreira, M.J., Wagner, T., Rigonatti, S.P., Castro, A.W., Souza, D.R., Riberto, M., Freedman, S.D., et al. (2006). A sham-controlled, phase II trial of transcranial direct current stimulation for the treatment of central pain in traumatic spinal cord injury. Pain 122: 197-209.

Gao, K.M., Lao, J., Guan, W.J., and Hu, J.J. (2018). Is it necessary to use the entire root as a donor when transferring contralateral C(7) nerve to repair median nerve? Neural. Regen. Res. 13: 94-99.

Gao, K.M., Lao, J., Zhao, X., and Gu, Y.D. (2010). Long-term outcome of selective contralateral C7 nerve transfer. Chin. J. Hand. Surg. 26: 324-327.

Gilbert, A., Pivato, G., and Kheiralla, T. (2006). Long-term results of primary repair of brachial plexus lesions in children. Microsurgery 26: 334-342.

Gu, Y.D. (1997). Functional motor innervation of brachial plexus roots. An intraoperative electrophysiological study. J. Hand. Surg. Br 22: $258-260$.

Gu, Y.D., Chen, D.S., Zhang, G.M., Cheng, X.M., Xu, J.G., Zhang, L.Y., Cai, P.Q., and Chen, L. (1998). Long-term functional results of contralateral C7 transfer. J. Reconstr. Microsurg. 14: 57-59.

Gu, Y.D. and Shen, L.Y. (1994). Electrophysiological changes after severance of the C7 nerve root. J. Hand. Surg. Br. 19: 69-71.

Gu, Y.D., Zhang, G.M., Chen, D.S., Yan, J.G., Cheng, X.M., and Chen, L. (1992). Seventh cervical nerve root transfer from the contralateral healthy side for treatment of brachial plexus root avulsion. J. Hand. Surg. Br. 17: 518-521.

Guan, J., Lin, J., Guan, X., and Jin, Q. (2019). Treatment of central paralysis of upper extremity using contralateral C7 nerve transfer via posterior spinal route. World Neurosurg. 125: 228-233.

Guan, J., Lin, J., Guan, X., Jin, Q., Chen, L., Shan, Q., Wu, J., Cai, X., Zhang, D., Tao, W., et al. (2020). Preliminary results of posterior contralateral cervical 7 nerve transposition in the treatment of upper limb plegia after a stroke. Brain Behav. 10: e01821.

Guan, J., Lin, J., Guan, X., Jin, Q., and Zhang, W. (2021). Contralateral C7 nerve transfer through posterior vertebral approach combined with selective posterior rhizotomy of the affected cervical nerve in the treatment of central upper limb spastic paralysis: a case report. Medicine (Baltim.) 100: e25061.

Guo, J., Zhao, X., Lao, J., and Gao, K. (2021). Why it is necessary to use the entire root rather than partial root when doing contralateral C7 nerve transfer: cortical plasticity also matters besides the amount of nerve fibers. Neural Plast. 2021: 8819380.

Hagg, T. (2006). Collateral sprouting as a target for improved function after spinal cord injury. J. Neurotrauma 23: 281-294.

Hankey, G.J. (2017). Stroke. Lancet 389: 641-654.

Hayashi, T. and Yokoi, A. (2016). Visuomotor map determines how visually guided reaching movements are corrected within and across trials. eNeuro 3, https://doi.org/10.1523/ENEURO.003216.2016

Hierner, R. and Berger, A.K. (2007). Did the partial contralateral C7-transfer fulfil our expectations? Results after 5 year experience. Acta Neurochir. Suppl. 100: 33-35.

Hosomi, K., Seymour, B., and Saitoh, Y. (2015). Modulating the pain network -neurostimulation for central poststroke pain. Nat. Rev. Neurol. 11: 290-299.

Hsieh, J.C., Cheng, H., Hsieh, H.M., Liao, K.K., Wu, Y.T., Yeh, T.C., and Ho, L.T. (2002). Loss of interhemispheric inhibition on the ipsilateral primary sensorimotor cortex in patients with brachial plexus injury: fMRI study. Ann. Neurol. 51: 381-385.

Hua, X.Y., Li, Z.Y., Xu, W.D., Zheng, M.X., Xu, J.G., and Gu, Y.D. (2012a). Interhemispheric functional reorganization after cross nerve transfer: via cortical or subcortical connectivity? Brain Res. 1471: 93-101.

Hua, X.Y., Liu, B., Qiu, Y.Q., Tang, W.J., Xu, W.D., Liu, H.Q., Xu, J.G., and $\mathrm{Gu}$, Y.D. (2013). Long-term ongoing cortical remodeling after contralateral C-7 nerve transfer. J. Neurosurg. 118: 725-729.

Hua, X.Y., Qiu, Y.Q., Li, T., Zheng, M.X., Shen, Y.D., Jiang, S., Xu, J.G., $\mathrm{Gu}, Y . D$. , and Xu, W.D. (2015). Contralateral peripheral neurotization for hemiplegic upper extremity after central neurologic injury. Neurosurgery 76: 187-195, discussion 195.

Hua, X.Y., Qiu, Y.Q., Wang, M., Zheng, M.X., Li, T., Shen, Y.D., Jiang, S., Xu, J.G., Gu, Y.D., Tsien, J., et al. (2016). Enhancement of contralesional motor control promotes locomotor recovery after unilateral brain lesion. Sci. Rep. 6: 18784.

Hua, X.Y., Zuo, C.T., Xu, W.D., Liu, H.Q., Zheng, M.X., Xu, J.G., and Gu, Y.D. (2012b). Reversion of transcallosal interhemispheric neuronal inhibition on motor cortex after contralateral C7 neurotization. Clin. Neurol. Neurosurg. 114: 1035-1038.

Hummel, F.C., Celnik, P., Pascual-Leone, A., Fregni, F., Byblow, W.D., Buetefisch, C.M., Rothwell, J., Cohen, L.G., and Gerloff, C. (2008). Controversy: noninvasive and invasive cortical stimulation show efficacy in treating stroke patients. Brain Stimul. 1: 370-382.

Jaillard, A., Martin, C.D., Garambois, K., Lebas, J.F., and Hommel, M. (2005). Vicarious function within the human primary motor cortex? A longitudinal fMRI stroke study. Brain 128: 1122-1138.

Jankowska, E. and Edgley, S.A. (2006). How can corticospinal tract neurons contribute to ipsilateral movements? A question with implications for recovery of motor functions. Neuroscientist 12: 67-79.

Jiang, S., Chen, W., Shen, Y.D., Qiu, Y.Q., Yu, A.P., and Xu, W.D. (2019). $\mathrm{C} 7$ transfer in a posterior intradural approach for treating 
hemiplegic upper-limbs: hypothesis and a cadaver feasibility study. Br. J. Neurosurg. 33: 413-417.

Jiang, S., Ichihara, S., Prunières, G., Peterson, B., Facca, S., Xu, W.D., and Liverneaux, P. (2016). Robot-assisted C7 nerve root transfer from the contralateral healthy side: a preliminary cadaver study. Hand Surg. Rehabil. 35: 95-99.

Jiang, S., Li, Z.Y., Hua, X.Y., Xu, W.D., Xu, J.G., and Gu, Y.D. (2010). Reorganization in motor cortex after brachial plexus avulsion injury and repair with the contralateral $\mathrm{C} 7$ root transfer in rats. Microsurgery 30: 314-320.

Jones, E.G. and Peters, A. (1986). Cerebral cortex. New York: Plenum 5, pp. 291-353.

Kachramanoglou, C., Li, D., Andrews, P., East, C., Carlstedt, T., Raisman, G., and Choi, D. (2011). Novel strategies in brachial plexus repair after traumatic avulsion. Br. J. Neurosurg. 25: $16-27$.

Kaiser, R., Waldauf, P., Ullas, G., and Krajcová, A. (2020). Epidemiology, etiology, and types of severe adult brachial plexus injuries requiring surgical repair: systematic review and metaanalysis. Neurosurg. Rev. 43: 443-452.

Kang, N., Summers, J.J., and Cauraugh, J.H. (2016). Transcranial direct current stimulation facilitates motor learning post-stroke: a systematic review and meta-analysis. J. Neurol. Neurosurg. Psychiatry 87: 345-355.

Kanning, K.C., Kaplan, A., and Henderson, C.E. (2010). Motor neuron diversity in development and disease. Annu. Rev. Neurosci. 33: 409-440.

Knotkova, H., Nitsche, M.A., and Cruciani, R.A. (2013). Putative physiological mechanisms underlying tDCS analgesic effects. Front. Hum. Neurosci. 7: 628.

Kosiyatrakul, A., Jitprapaikulsarn, S., Durand, S., and Oberlin, C. (2009). Recovery of brachial plexus injury after shoulder dislocation. Injury 40: 1327-1329.

Kuang, R.Z. and Kalil, K. (1990). Specificity of corticospinal axon arbors sprouting into denervated contralateral spinal cord. J. Comp. Neurol. 302: 461-472.

Lanaras, T.I., Schaller, H.E., and Sinis, N. (2009). Brachial plexus lesions: 10 years of experience in a center for microsurgery in Germany. Microsurgery 29: 87-94.

Leblebicioglu, G., Ayhan, C., Firat, T., Uzumcugil, A., Yorubulut, M., and Doral, M.N. (2016). Recovery of upper extremity function following endoscopically assisted contralateral C7 transfer for obstetrical brachial plexus injury. J. Hand Surg. Eur. 41: 863-874.

Lefaucheur, J.P. (2016). Cortical neurostimulation for neuropathic pain: state of the art and perspectives. Pain 157: S81-s89.

Lefaucheur, J.P., Drouot, X., Keravel, Y., and Nguyen, J.P. (2001). Pain relief induced by repetitive transcranial magnetic stimulation of precentral cortex. Neuroreport 12: 2963-2965.

Lefebvre, S. and Liew, S.L. (2017). Anatomical parameters of tDCS to modulate the motor system after stroke: a review. Front. Neurol. 8: 29.

Lemon, R.N. (2008). Descending pathways in motor control. Annu. Rev. Neurosci. 31: 195-218.

Li, H., Yang, J.T., Tan, W.P., Zhu, Y., and Xiang, Q.S. (2012). The anatomical study of contralateral $C 7$ transfer through the prespinal and the posterior cervical route. Prog. Mod. Biomed. 12: 2054-2056.

Li, P., Shen, Y., Xu, J., Liang, C., Jiang, S., Qiu, Y., Yin, H., Feng, J., Li, T., Shen, J., et al. (2020). Contralateral cervical seventh nerve transfer for spastic arm paralysis via a modified prespinal route: a cadaveric study. Acta Neurochir. 162: 141-146.

Li, R., Machol, J.A.t., Liu, X., Hettinger, P.C., Flugstad, N.A., Yan, J.G., Matloub, H.S., and Hyde, J.S. (2014). C7 nerve root sensory distribution in peripheral nerves: a bold functional magnetic resonance imaging investigation at 9.4 T. Muscle Nerve 49: 40-46.

Li, T., Hua, X.Y., Zheng, M.X., Wang, W.W., Xu, J.G., Gu, Y.D., and Xu, W.D. (2015a). Different cerebral plasticity of intrinsic and extrinsic hand muscles after peripheral neurotization in a patient with brachial plexus injury: a TMS and fMRI study. Neurosci. Lett. 604: 140-144.

Li, W., Wang, S., Zhao, J., Rahman, M.F., Li, Y., Li, P., and Xue, Y. (2015b). Complications of contralateral C-7 transfer through the modified prespinal route for repairing brachial plexus root avulsion injury: a retrospective study of 425 patients. J. Neurosurg. 122: 1421-1428.

Lindblom, U.F. and Ottosson, J.O. (1957). Influence of pyramidal stimulation upon the relay of coarse cutaneous afferents in the dorsal horn. Acta Physiol. Scand. 38: 309-318.

Liu, Y., Hegarty, S., Winter, C., Wang, F., and He, Z. (2020). Viral vectors for neuronal cell type-specific visualization and manipulations. Curr. Opin. Neurobiol. 63: 67-76.

Liu, Y., Yang, X., Gao, K., Yu, H., Xiao, F., Zhuang, Y., and Lao, J. (2018). Outcome of contralateral $\mathrm{C} 7$ transfers to different recipient nerves after global brachial plexus avulsion. Brain Behav. 8: e01174.

Lotze, M., Markert, J., Sauseng, P., Hoppe, J., Plewnia, C., and Gerloff, C. (2006). The role of multiple contralesional motor areas for complex hand movements after internal capsular lesion. J. Neurosci. 26: 6096-6102.

Lou, L., Shou, T., Li, Z., Li, W., and Gu, Y. (2006). Transhemispheric functional reorganization of the motor cortex induced by the peripheral contralateral nerve transfer to the injured arm. Neuroscience 138: 1225-1231.

Ma, H., Zheng, M., Lu, Y., Hua, X., and Xu, W. (2018). Cerebral plasticity after contralateral cervical nerve transfer in human by longitudinal PET evaluation. J. Clin. Neurosci. 48: 95-99.

Maarrawi, J., Mertens, P., Luaute, J., Vial, C., Chardonnet, N., Cosson, M., and Sindou, M. (2006). Long-term functional results of selective peripheral neurotomy for the treatment of spastic upper limb: prospective study in 31 patients. J. Neurosurg. 104: 215-225.

McGuiness, C.N. and Kay, S.P. (2002). The prespinal route in contralateral C7 nerve root transfer for brachial plexus avulsion injuries. J. Hand Surg. Br. 27: 159-160.

Midrio, M. (2006). The denervated muscle: facts and hypotheses. A historical review. Eur. J. Appl. Physiol. 98: 1-21.

Morishita, T., Hyakutake, K., Saita, K., Takahara, M., Shiota, E., and Inoue, T. (2015). Pain reduction associated with improved functional interhemispheric balance following transcranial direct current stimulation for post-stroke central pain: a case study. J. Neurol. Sci. 358: 484-485.

Morishita, T. and Inoue, T. (2016). Brain stimulation therapy for central post-stroke pain from a perspective of interhemispheric neural network remodeling. Front. Hum. Neurosci. 10: 166.

Murase, N., Duque, J., Mazzocchio, R., and Cohen, L.G. (2004). Influence of interhemispheric interactions on motor function in chronic stroke. Ann. Neurol. 55: 400-409.

Nahmias, F., Debes, C., de Andrade, D.C., Mhalla, A., and Bouhassira, D. (2009). Diffuse analgesic effects of unilateral repetitive 
transcranial magnetic stimulation (rTMS) in healthy volunteers. Pain 147: 224-232.

Narakas, A.O. and Hentz, V.R. (1988). Neurotization in brachial plexus injuries. Indication and results. Clin. Orthop. Relat. Res.: 43-56, https://doi.org/10.1097/00003086-198812000-00008.

Netz, J., Lammers, T., and Hömberg, V. (1997). Reorganization of motor output in the non-affected hemisphere after stroke. Brain 120: 1579-1586.

Nguyen, J.P., Nizard, J., Keravel, Y., and Lefaucheur, J.P. (2011). Invasive brain stimulation for the treatment of neuropathic pain. Nat. Rev. Neurol. 7: 699-709.

Nicolas, N., Kobaiter-Maarrawi, S., Georges, S., Abadjian, G., and Maarrawi, J. (2018). Motor cortex stimulation regenerative effects in peripheral nerve injury: an experimental rat model. World Neurosurg. 114: e800-e808.

Nowak, D.A., Grefkes, C., Ameli, M., and Fink, G.R. (2009). Interhemispheric competition after stroke: brain stimulation to enhance recovery of function of the affected hand. Neurorehabil. Neural Repair 23: 641-656.

Pan, F., Chen, L., Ding, F., Zhang, J., and Gu, Y.D. (2015). Expression profiles of MiRNAs for intrinsic musculature of the forepaw and biceps in the rat model simulating irreversible muscular atrophy of obstetric brachial plexus palsy. Gene 565: 268-274.

Pan, F., Wei, H.F., Chen, L., and Gu, Y.D. (2012). Different functional reorganization of motor cortex after transfer of the contralateral $\mathrm{C} 7$ to different recipient nerves in young rats with total brachial plexus root avulsion. Neurosci. Lett. 531: 188-192.

Pan, X., Zhao, G., Yang, X., Hua, Y., Wang, J., Ying, Q., and Mi, J. (2020). Contralateral $\mathrm{C} 7$ nerve transfer via the prespinal route in treatment of spastic paralysis of upper limb after cerebral palsy. Br. J. Neurosurg.: 1-5, https://doi.org/10.1080/02688697. 2020.1859091.

Passard, A., Attal, N., Benadhira, R., Brasseur, L., Saba, G., Sichere, P., Perrot, S., Januel, D., and Bouhassira, D. (2007). Effects of unilateral repetitive transcranial magnetic stimulation of the motor cortex on chronic widespread pain in fibromyalgia. Brain 130: 2661-2670.

Peng, F., Cai, P.Q., Chen, D.S., Chen, L., and Gu, Y.D. (2003). Modified procedure of contralateral $C 7$ transfer for repair of roots and trunks injuries of brachial plexus. Chin. J. Hand Surg. 19: 66-68.

Petuchowksi, J., Kieras, K., and Stein, K. (2018). Rehabilitation strategies following surgical treatment of upper extremity spasticity. Hand Clin. 34: 567-582.

Post, R., de Boer, K.S., and Malessy, M.J. (2012). Outcome following nerve repair of high isolated clean sharp injuries of the ulnar nerve. PLoS One 7: e47928.

Qin, B., Gu, L., Xiang, J., Fu, G., Qi, J., Wang, H., Zhang, D., Zheng, J., Liu, X., and Zhu, J. (2012). Elementary research of constructive feature and three-dimensional reconstruction of nerve bundles of $\mathrm{C} 7$ anterior and posterior division end. Zhongguo Xiu Fu Chong Jian Wai Ke Za Zhi 26: 97-101.

Rubiano, A.M., Carney, N., Chesnut, R., and Puyana, J.C. (2015). Global neurotrauma research challenges and opportunities. Nature 527: S193-S197.

Rudomin, P. (1990). Presynaptic inhibition of muscle spindle and tendon organ afferents in the mammalian spinal cord. Trends Neurosci. 13: 499-505.

Sammer, D.M., Kircher, M.F., Bishop, A.T., Spinner, R.J., and Shin, A.Y. (2012). Hemi-contralateral C7 transfer in traumatic brachial plexus injuries: outcomes and complications. J. Bone Joint Surg. Am. 94: 131-137.

Seddon, H.J. (1972). Surgical disorders of the peripheral nerves. Baltimore: Williams \& Wilkins.

Sindou, M. and Mertens, P. (1988). Selective neurotomy of the tibial nerve for treatment of the spastic foot. Neurosurgery 23 : 738-744.

Sindou, M.P., Simon, F., Mertens, P., and Decq, P. (2007). Selective peripheral neurotomy (SPN) for spasticity in childhood. Childs Nerv. Syst. 23: 957-970.

Singer, J., Conigliaro, A., Spina, E., Law, S.W., and Levine, S.R. (2017). Central poststroke pain: a systematic review. Int. J. Stroke 12: 343-355.

Songcharoen, P. (2008). Management of brachial plexus injury in adults. Scand. J. Surg. 97: 317-323.

Steyerberg, E.W., Wiegers, E., Sewalt, C., Buki, A., Citerio, G., De Keyser, V., Ercole, A., Kunzmann, K., Lanyon, L., Lecky, F., et al. (2019). Case-mix, care pathways, and outcomes in patients with traumatic brain injury in CENTER-TBI: a European prospective, multicentre, longitudinal, cohort study. Lancet Neurol. 18: 923-934.

Sun, G., Wu, Z., Wang, X., Tan, X., and Gu, Y. (2014). Nerve transfer helps repair brachial plexus injury by increasing cerebral cortical plasticity. Neural Regen. Res. 9: 2111-2114.

Synnot, A., Chau, M., Pitt, V., O’Connor, D., Gruen, R.L., Wasiak, J., Clavisi, O., Pattuwage, L., and Phillips, K. (2017). Interventions for managing skeletal muscle spasticity following traumatic brain injury. Cochrane Database Syst. Rev. 11, Cd008929.

Tantigate, D., Wongtrakul, S., Vathana, T., Limthongthang, R., and Songcharoen, P. (2015). Neuropathic pain in brachial plexus injury. Hand Surg. 20: 39-45.

Terzis, J.K. (1987). Microreconstruction of nerve injuries. Philadelphia, USA): Saunders, pp. 285-324.

Terzis, J.K. and Kokkalis, Z.T. (2009). Selective contralateral c7 transfer in posttraumatic brachial plexus injuries: a report of 56 cases. Plast. Reconstr. Surg. 123: 927-938.

Terzis, J.K. and Kostopoulos, V.K. (2007). The surgical treatment of brachial plexus injuries in adults. Plast. Reconstr. Surg. 119: $73 e-92 e$.

Treede, R.D., Jensen, T.S., Campbell, J.N., Cruccu, G., Dostrovsky, J.O., Griffin, J.W., Hansson, P., Hughes, R., Nurmikko, T., and Serra, J. (2008). Neuropathic pain: redefinition and a grading system for clinical and research purposes. Neurology 70: 1630-1635.

Tsubokawa, T., Katayama, Y., Yamamoto, T., Hirayama, T., and Koyama, S. (1991). Chronic motor cortex stimulation for the treatment of central pain. Acta Neurochir. Suppl. 52: 137-139.

Tsubokawa, T., Katayama, Y., Yamamoto, T., Hirayama, T., and Koyama, S. (1993). Chronic motor cortex stimulation in patients with thalamic pain. J. Neurosurg. 78: 393-401.

Tu, Y.K., Tsai, Y.J., Chang, C.H., Su, F.C., Hsiao, C.K., and Tan, J.S. (2014). Surgical treatment for total root avulsion type brachial plexus injuries by neurotization: a prospective comparison study between total and hemicontralateral $\mathrm{C} 7$ nerve root transfer. Microsurgery 34: 91-101.

Twitchell, T.E. (1951). The restoration of motor function following hemiplegia in man. Brain 74: 443-480.

Vanaclocha, V., Herrera, J.M., Verdu-Lopez, F., Gozalbes, L., SanchezPardo, M., Rivera, M., Martinez-Gomez, D., and Mayorga, J.D. (2015). Transdiscal C6-C7 contralateral C7 nerve root transfer in 
the surgical repair of brachial plexus avulsion injuries. Acta Neurochir. 157: 2161-2167.

Vorobiev, V., Govoni, P., Rizzolatti, G., Matelli, M., and Luppino, G. (1998). Parcellation of human mesial area 6: cytoarchitectonic evidence for three separate areas. Eur. J. Neurosci. 10: 2199-2203.

Waikakul, S., Orapin, S., and Vanadurongwan, V. (1999). Clinical results of contralateral $C 7$ root neurotization to the median nerve in brachial plexus injuries with total root avulsions. J. Hand Surg. Br. 24: 556-560.

Wang, G.B., Yu, A.P., Ng, C.Y., Lei, G.W., Wang, X.M., Qiu, Y.Q., Feng, J.T., Li, T., Chen, Q.Z., He, Q.R., et al. (2018). Contralateral C7 to $C 7$ nerve root transfer in reconstruction for treatment of total brachial plexus palsy: anatomical basis and preliminary clinical results. J. Neurosurg. Spine 29: 491-499.

Wang, L., Zhao, X., Gao, K., Lao, J., and Gu, Y.D. (2011). Reinnervation of thenar muscle after repair of total brachial plexus avulsion injury with contralateral $\mathrm{C} 7$ root transfer: report of five cases. Microsurgery 31: 323-326.

Wang, M., Li, Z.Y., Xu, W.D., Hua, X.Y., Xu, J.G., and Gu, Y.D. (2010). Sensory restoration in cortical level after a contralateral $C 7$ nerve transfer to an injured arm in rats. Neurosurgery 67: 136-143, discussion 143.

Wang, S.F., Hu, Q., Wang, H.H., Su, Y.N., Zhao, J.H., and Chang, W.S. (2003). The anatomical and clinical study of contralateral C7 transfer through the prespinal route. Chin. J. Hand Surg. 19: 69-71.

Wang, S.F., Li, P.C., Xue, Y.H., Yiu, H.W., Li, Y.C., and Wang, H.H. (2013). Contralateral C7 nerve transfer with direct coaptation to restore lower trunk function after traumatic brachial plexus avulsion. J. Bone Joint Surg. Am. 95: 821-827, s821-822.

Wang, S.F., Wang, H.H., Su, Y.N., Pan, Y.W., Chen, S.L., Hu, Q., and Zhao, J.H. (2004). The clinical study of healthy $C 7$ nerve root transfer to repair the brachial plexus avulsion through the anterior spinal approach. Chin. J. Orthop. 24: 453-456.

Wang, S.F. and Xue, Y.H. (2014). Contralateral C7 nerve transfer with direct coaptation to restore lower trunk function after traumatic brachial plexus avulsion injuries: surgical technique. JBJS Essent Surg. Tech. 4: e5.

Wang, W.W., Liu, B., Hua, X.Y., Liu, H.Q., Tang, W.J., Zhang, J.H., Sun, H.P., and feng, X.Y. (2015). Motor imagery of the patients with brachial plexus injury before and after contralateral C7 nerve transfer: an fMRI study. Chin. Comput. Med. Imag. 21: 6-10.

Wang, X., Liu, Y., Li, X., Zhang, Z., Yang, H., Zhang, Y., Williams, P.R., Alwahab, N.S.A., Kapur, K., Yu, B., et al. (2017). Deconstruction of corticospinal circuits for goal-directed motor skills. Cell 171: 440-455, e414.

Wang, Y.F., Wang, B., Li, F., Zhu, Z., Li, Y.Q., Su, L., and Zou, S.W. (2009). The anatomical study of contralateral C7 transfer through the vertebral body route. Chin. J. Microsurg. 32: 133-135.

Welniarz, Q., Dusart, I., and Roze, E. (2017). The corticospinal tract: evolution, development, and human disorders. Dev. Neurobiol. 77: 810-829.

Wise, S.P., Boussaoud, D., Johnson, P.B., and Caminiti, R. (1997). Premotor and parietal cortex: corticocortical connectivity and combinatorial computations. Annu. Rev. Neurosci. 20: 25-42.

Wolpert, D.M. and Flanagan, J.R. (2010). Motor learning. Curr. Biol. 20: R467-72.
Wu, D., Qian, L., Zorowitz, R.D., Zhang, L., Qu, Y., and Yuan, Y. (2013). Effects on decreasing upper-limb poststroke muscle tone using transcranial direct current stimulation: a randomized shamcontrolled study. Arch. Phys. Med. Rehabil. 94: 1-8.

Wu, J.X., Chen, L., Ding, F., Chen, L.Z., and Gu, Y.D. (2016). mRNA expression characteristics are different in irreversibly atrophic intrinsic muscles of the forepaw compared with reversibly atrophic biceps in a rat model of obstetric brachial plexus palsy (OBPP). J. Muscle Res. Cell Motil. 37: 17-25.

Xia, C.L., Jing, Y., Tian, Y., Wang, X., Han, Q., Wang, Y., Han, Y.Y., Su, L., Wang, Y.F., and Li, Y.Q. (2010). Feasilibity of contralateral C7 transfer for treatment of brachial plexus root avulsions through C7 vertebrae channel. J. Jilin Univ. Med. Ed. 36: 727-730.

Xiang, Q.S., Yang, J.T., Liu, G.L., Tan, W.P., Li, H., and Zhang, S.S. (2012). Anatomical study on contralateral C7 nerve transfer via posterior spinal route for treatment of brachial plexus root avulsion injury. Chin. J. Reparat. Reconstruct. Surg. 26: 235-237.

Xu, J.G., Hu, S.N., Wang, H., and Gu, Y.D. (1996). Histochemical study on $\mathrm{C} 7$ roots and its clinical significance. Chin. J. Clin. Anat. 14: 243-245.

Xu, J.G., Wang, H., Hu, S.N., and Gu, Y.D. (2004). Selective transfer of the $\mathrm{C} 7$ nerve root: an experimental study. J. Reconstr. Microsurg. 20: 463-470, discussion 471-462.

Xu, L., Gu, Y., Xu, J., Lin, S., Chen, L., and Lu, J. (2008). Contralateral C7 transfer via the prespinal and retropharyngeal route to repair brachial plexus root avulsion: a preliminary report. Neurosurgery 63: 553-558, discussion 558-559.

Xu, L., Gu, Y.D., Xu, J.G., Xu, W.D., Lu, J.Z., Lin, S., Shen, Y.D., Shen, H., and Han, D. (2007). The contralateral C7 transfer via prespinal route to repair the brachial plexus avulsion: a preliminary study on its clinical effect. Chin. J. Microsurg. 30: 270-273.

$X u$, W.D. (2020). Surgical technique of Xu's CC7 procedure "contralateral $\mathrm{C} 7$ to $\mathrm{C} 7$ cross nerve transfer through a trans longus colli, prespinal route for treating spastic arm". Oper. Neurosurg. (Hagerstown) 20: 61-68.

Xu, W.D., Hua, X.Y., Zheng, M.X., Xu, J.G., and Gu, Y.D. (2011). Contralateral $C 7$ nerve root transfer in treatment of cerebral palsy in a child: case report. Microsurgery 31: 404-408.

Yang, G., Chang, K.W., and Chung, K.C. (2015). A systematic review of contralateral C7 transfer for the treatment of traumatic brachial plexus injury: Part 1. Overall outcomes. Plast. Reconstr. Surg. 136: 794-809.

Yang, K., Jiang, F., Zhang, S., Zhao, H., Shi, Z., Liu, J., and Cao, X. (2020). Extradural contralateral $C 7$ nerve root transfer in a cervical posterior approach for treating spastic limb paralysis: a cadaver feasibility study. Spine 45: E608-15.

Yang, X., Liu, Y., Zhao, X., and Lao, J. (2018). Electrophysiologic recovery of the abductor pollicis brevis after contralateral $C 7$ nerve transfer in 95 patients with global brachial plexus avulsion. J. Electromyogr. Kinesiol. 43: 158-161.

Yang, Y., Wang, S.F., Su, H.P., Xue, Y.H., Li, W.J., and Li, Y.C. (2017). Contralateral $C 7$ nerve transfer via a modified prespinal route in repair of brachial plexus avulsion injuries. Chin. J. Hand Surg. 33: 32-35.

Yu, A., Wang, S., Cheng, X., Liang, W., Bai, R., Xue, Y., and Li, W. (2017). Functional connectivity of motor cortical network in patients with brachial plexus avulsion injury after contralateral cervical nerve transfer: a resting-state fMRI study. Neuroradiology 59: 247-253. 
Yu, A.P., Jiang, S., Zhao, H.L., Liang, Z.H., Qiu, Y.Q., Shen, Y.D., Wang, G.B., Liang, C., and Xu, W.D. (2019). Application of CUBE-STIR MRI and high-frequency ultrasound in contralateral cervical 7 nerve transfer surgery. Br. J. Neurosurg.: 1-6, https://doi.org/10.1080/02688697.2019.1584661.

Yu, Z.J., Sui, S., Yu, S., Huang, Y., and Sheng, J. (2003). Contralateral normal C7 nerve transfer after upper arm shortening for the treatment of total root avulsion of the brachial plexus: a preliminary report. Plast. Reconstr. Surg. 111: 1465-1469.

Zhang, C.G. and Gu, Y.D. (2011). Contralateral C7 nerve transfer - our experiences over past 25 years. J Brachial Plex. Peripher. Nerve Inj. 6: 10
Zheng, M.X., Hua, X.Y., Feng, J.T., Li, T., Lu, Y.C., Shen, Y.D., Cao, X.H., Zhao, N.Q., Lyu, J.Y., Xu, J.G., et al. (2018). Trial of contralateral seventh cervical nerve transfer for spastic arm paralysis. N. Engl. J. Med. 378: 22-34.

Ziemann, U., Ishii, K., Borgheresi, A., Yaseen, Z., Battaglia, F., Hallett, M., Cincotta, M., and Wassermann, E.M. (1999). Dissociation of the pathways mediating ipsilateral and contralateral motorevoked potentials in human hand and arm muscles. J. Physiol. 518: 895-906.

Zuo, C.T., Hua, X.Y., Guan, Y.H., Xu, W.D., Xu, J.G., and Gu, Y.D. (2010). Long-range plasticity between intact hemispheres after contralateral cervical nerve transfer in humans. J. Neurosurg. 113: 133-140. 